\title{
The crystal structure of rathite-I*
}

\author{
By F. Marumo and W. NowackI \\ Abteilung für Kristallographie und Strukturlehre, \\ Mineralogisches Institut, Universität Bern, Schweiz
}

(Received May 10, 1965)

\begin{abstract}
Auszug
Die Kristallstruktur von Rathit-I wurde mittels dreidimensionaler Intensitätsdaten bestimmt. Vier Formeleinhoiten $(\mathbf{P b}, \mathbf{T l})_{2} \mathbf{A s}_{4}(\mathbf{A s}, \mathbf{A g}) \mathbf{S}_{10}$ sind in der Einheitszelle der Symmetrie $P 2_{1} / a$ mit $a=25,16 \AA, b=7,94 \AA, c=8,47 \AA$, $\beta=100^{\circ} 28^{\prime}$ enthalten. Die wahre Symmetrie von Rathit-I ist möglicherweise triklin. Die Lösung lieferten die Āhnlichkeit der Struktur mit derjenigen von Rathit-III und spezielle Verhältnisse der Röntgendiagramme.

Von drei unabhängigen $\mathrm{Pb}(\mathrm{T})$-Atomen sind zwei von neun S-Atomen umgeben, das andere von sieben. Die As-Atome weisen trigonal-pyramidale Koordination durch die S-Atome auf. Von einem As-Atom wird angenommen, daß es statistisch von zwei verschiedenen trigonal-pyramidalen S-Koordinationen umgeben wird. Ein anderes As-Atom ist teilweise durch Ag ersetzt.

Die Struktur besteht aus zweierlei Schichten parallel zu (100). Die erste Art hat die Zusammensetzung $(\mathrm{Pb}, \mathrm{T}) \mathrm{S}_{3}$ und besteht aus den Koordinationspoly. edern um die $\mathrm{Pb}(\mathrm{Tl})$-Atome mit Neuner-Koordination. Die zweite Art ist aus $\mathrm{Pb}(\mathrm{Tl})$-, $\mathrm{As}(\mathrm{Ag})$ - und S-Atomen zusemmengesetzt, welche ein deformiertes PbS-Gitter bilden. Trigonale As-S $\mathrm{S}_{\mathbf{8}}$-Pyramiden sind zu Ketten endlicher Länge vereinigt.
\end{abstract}

\section{Abstract}

The crystal structure of rathite-I has been determined with the use of three-dimensional intensity data. Four chemical units of $(\mathrm{Pb}, \mathrm{Tl})_{3} \mathbf{A s}_{\mathbf{4}}(\mathbf{A s}, \mathbf{A g}) \mathrm{S}_{10}$ are contained in the unit-cell of the symmetry $P 2_{1} / a$ with $a=25.16 \AA, b=$ $7.94 \AA, c=8.47 \AA, \beta=100^{\circ} 28^{\prime}$. The true symmetry of rathite-I may be triclinic. The solution was obtained from the similarity of the crystal structure to that of rathite-III and from a peculiar feature of the x-ray diagrams.

Among three independent $\mathrm{Pb}(\mathrm{Tl})$ atoms two are surrounded by nine $\mathrm{S}$ atoms and the other is surrounded by seven $S$ atoms. As atoms have trigonal-pyramidal coordinations by $S$ atoms. One As atom, however, is believed to occupy statistically two different trigonal-pyramidal S coordinations. Another As atom is partially replaced by Ag.

* Contribution No. 155. - Part 19 on sulfides and sulfosalts. 
The structure is composed of two kinds of layers parallel to (100). Layers of the first kind have the composition $(\mathrm{Pb}, \mathrm{Tl}) \mathrm{S}_{3}$, and consist of coordination poly. hedra around the $\mathrm{Pb}(\mathrm{Tl})$ atoms which are coordinated by nine $\mathrm{S}$ atoms. The layers of the second kind are composed of $\mathrm{Pb}(\mathrm{Tl}), \mathrm{As}(\mathrm{Ag})$ and $\mathrm{S}$ atoms, having a deformed PbS-type structure. Trigonal As- $\mathrm{S}_{3}$ pyramids are linked into strings of finite length.

\section{Introduction}

Rathite-I, $(\mathrm{Pb}, \mathrm{Tl})_{3} \mathrm{As}_{4}(\mathrm{As}, \mathrm{Ag}) \mathrm{S}_{10}$, is a mineral of a sulfosalt group, to which rathite-II, rathite-III, rathite-IV, dufrenoysite, baumhauerite and scleroclase belong. A characteristic feature of these minerals is that they have periods of $8.4 \AA$ and $7.9 \AA$ along two mutually perpendicular directions. Though most of the structures have already been investigated, no precise structure has yet been revealed owing to the large absorption effects and the large unit-cell dimensions. Some of the structures reported contain unreasonable features such as, for example, infinite chains of As-S $\mathrm{S}_{3}$ pyramids along the $8.4 \AA$ axes which, as was pointed out by Y. IrTAKA and W. NoWACKI (1961), cannot exist.

The structure determination of rathite-I was carried out in order to obtain precise information concerning the structural principles of this group of minerals. It was also desired to clarify the relationship of rathite-III and rathite-I, which are dimorphous if the small amount of $\mathrm{Tl}$ and $\mathrm{Ag}$ in the latter plays no significant role in the formation of the mineral and can be replaced by $\mathrm{Pb}$ and by As respectively.

Rathite-III (LE BIHAN, 1962) has hitherto not been found by us in the Lengenbach quarry. It is important to mention that the rathite-I of LE Brbax (1962) is almost identical with dufrenoysite and was called rathite-I $a$ by us (NowACKI et al., 1964). Rathite-II was first described by BrRpY (1953). The lattice constants, space group and chemical composition are:

\begin{tabular}{|c|c|c|c|c|c|c|}
\hline Mineral & Formula & $a$ & $b$ & $c$ & $\beta$ & Space group \\
\hline Rathite-I & $(\mathrm{Pb}, \mathrm{Tl})_{9} \mathrm{Ag}_{4}(\mathrm{As}, \mathrm{Ag}) \mathrm{S}_{10}$ & 25.16 & 7.94 & 8.47 & $100^{\circ} 28^{\prime}$ & $P 2_{1} / a(P I)$ \\
\hline Rathite-III & $\mathrm{Pb}_{8} \mathrm{As}_{5} \mathrm{~S}_{10}$ & 24.52 & 7.91 & 8.43 & $90^{\circ}$ & $P 2_{1}$ \\
\hline Rathite-II & $\mathrm{Pb}_{9} \mathrm{As}_{18} \mathrm{~S}_{28}$ & 8.43 & 70.9 & 7.91 & $90^{\circ}$ & $P 2_{1}$ \\
\hline
\end{tabular}

Thus, rathite-I and -III form two modifications of a single species and should perhaps have a name different from rathite-II; it is not, however, possible for us to introduce one. In the Lengenbach quarry rathite-II is frequently found, whereas rathite-I occurs rarely, and 
then usually polysynthetically twinned. The microprobe analysis (NowACKI und BAHEZRE, 1963) yielded the composition $\mathrm{Pb}=41.2 \pm 1$, $\mathrm{As}=27.0 \pm 0.5, \mathrm{~S}=28 \pm(1-2), \mathrm{Tl}=3.6 \pm 1, \Sigma=99.7 \%$.

\section{Experimental}

We looked through a large number of specimens from Lengenbach for a suitable rathite-I crystal as described by Peacock and BerRy (1940), but could not find one untwinned. Finally, through the kindness of Dr. L. G. BERRY (Queens University, Kingston, Canada) we obtained a good crystal (also from Lengenbach) for intensity measurements.

The unit-cell dimensions obtained from Weissenberg photographs are,

$$
\begin{aligned}
& a=25.16 \pm 0.02 \AA, \quad b=7.94 \pm 0.01 \AA, \quad c=8.47 \pm 0.01 \AA, \\
& \alpha=90^{\circ} \pm 10^{\prime}, \quad \beta=100^{\circ} 28^{\prime} \pm 10^{\prime}, \quad \gamma=90^{\circ} \pm 10^{\prime} \text {. }
\end{aligned}
$$

Although the space group of rathite-I was reported as $P 2_{1} / a$, the Weissenberg photographs showed small discrepancies between the intensities of $h k l$ and $\bar{h} k l$ reflections, indicating triclinic symmetry for this crystal. Moreover, several weak reflections with $h=$ odd were observed among the $h 0 l$ reflections. The true space group must, therefore, be $P 1$ or $P \overline{1}$. However, it is difficult to say whether these small deviations from monoclinic symmetry are common to all rathite-I crystals or whether they are only a special characteristic of the crystal examined, caused by a small content of $\mathrm{Tl}$ and Ag. For the structure determination the space group $P 2_{1} / a$ was assumed, and the average intensities of the $h k l$ and $\bar{h} k l$ reflections were used, the difference being very small.

A sphere with a radius of $0.06 \mathrm{~mm}$ was propared for the intensity measurement from a piece of the crystal. The integrated Weissenberg photographs were taken with $\mathrm{Cu} K \alpha$ radiation up to the 7-th layer around the $b$ axis and up to the second layer around the $c$ axis. The intensities were measured with a Joyce-Loebl microdensitometer, and corrected for the Lorentz-polarization and absorption effects with the programme of $\mathrm{Y}$. IITAKA for the Bull $\Gamma$ AET electronic computer. The linear absorption coefficient of the crystal is $855 \mathrm{~cm}^{-1}$ for $\mathrm{Cu} K \alpha$ and the absorption-correction factors for the sphere range between 180 at $\theta=0^{\circ}$ and 14 at $\theta=90^{\circ}$.

The chemical analysis of the crystal was carried out by W. NoWACKI and C. BAHEZRE (1963) with a Castaing x-ray microanalyser. 
The unit-cell content calculated from the result, assuming $5.37 \mathrm{~g} / \mathrm{cm}^{3}$ (DANA's system of mineralogy, Vol. I, 1944) for the density, is $\mathrm{Pb}_{10.7} \mathrm{Tl}_{0.9} \mathrm{As}_{19.3} \mathrm{~S}_{40.1}$, or approximately $\mathrm{Pb}_{11} \mathrm{Tl}_{1} \mathrm{As}_{20} \mathrm{~S}_{40}$. In the actual structure-factor calculations, the $\mathrm{Tl}$ atoms were taken as $\mathrm{Pb}$ atoms, since the differences between the atomic scattering factors of these two elements are quite small, and since the number of $\mathrm{Tl}$ atoms in the unit cell is less than the value required by the space group $P 2_{1} / a$.

\section{Structure analysis}

Since the $h k 0$ x-ray diffraction diagram of rathite-I is almost identical with that of rathite-III (M.-TH. LE BIHAN, 1962), the $c$ axis projection of the structure should have the same atomic arrangement as that of rathite-III. Actually the values $a \sin \beta, b$ and $c$ for rathite-I (24.75 $\AA, 7.94 \AA, 8.47 \AA$ ) are nearly equal to the values found for rathite-III (24.52 $\AA, 7.91 \AA, 8.43 \AA)$ and the chemical contents of their unit cells are identical if the $T /$ atoms in rathite-I are replaced by $\mathrm{Pb}$ atoms $\left(\mathrm{Pb}_{12} \mathrm{As}_{20} \mathrm{~S}_{40}\right)$. Calculation of the $h k 0$ structure factors were, therefore, carried out with a programme by Y. IITAkA for Bull $\Gamma$ AET utilizing the atomic coordinates of rathite-III; fairly good agreement between the observed and the calculated structure factors was obtained, the $R$-factor being 0.38 . This projection was refined by difference Fourier syntheses until the $R$-value was reduced to 0.16 .

The $z$ coordinates were obtained from a special feature of the $h 0 l$ x-ray diagram. Since the $h 0 l$ intensity distribution along the $c$ axis direction in reciprocal space is periodic to a fairly good approximation with the period 4, all atoms should lie nearly on a set of equally spaced planes perpendicular to the $c$ axis, the interplaner spacing being $c / 4$. There are two possible sets of planes which satisfy both this condition and the symmetry requirement for $P 2_{1} / a$ :

$z=\frac{1}{8}+\frac{x}{2}=\frac{1}{8}-x \cos \beta, z=\frac{3}{8}+\frac{x}{2}, z=\frac{5}{8}+\frac{x}{2}, z=\frac{7}{8}+\frac{x}{2}$, and

$$
z=\frac{x}{2}, z=\frac{1}{4}+\frac{x}{2}, z=\frac{2}{4}+\frac{x}{2}, z=\frac{3}{4}+\frac{x}{2} .
$$

The structural similarity to rathite-III as well as crystallochemical considerations suggested that the correct set should be the former, and furmished two probable models of the structure. The true structure was found after several cycles of refinements of these models tested with the $h 0 l$ difference Fourier projection. The $R$ value of the correct model was reduced from the initial value 0.49 to 0.19 for the $h 0 l$ reflections during the refinement. 


\section{Refinement}

During the preliminary study with two-dimensional data, it was found from the Fourier projections that the $\mathrm{As}(5)$ atom has a lower electron density than the other As atoms and that there is a peak at a position about $0.6 \AA$ apart from the position postulated for $\mathrm{As}(5)$. The agreement between the observed and the calculated structure factors becomes worse if $\mathrm{As}(5)$ is put at this peak. Therefore it was suspected that the $\mathrm{As}(5)$ atom statistically occupies both positions.

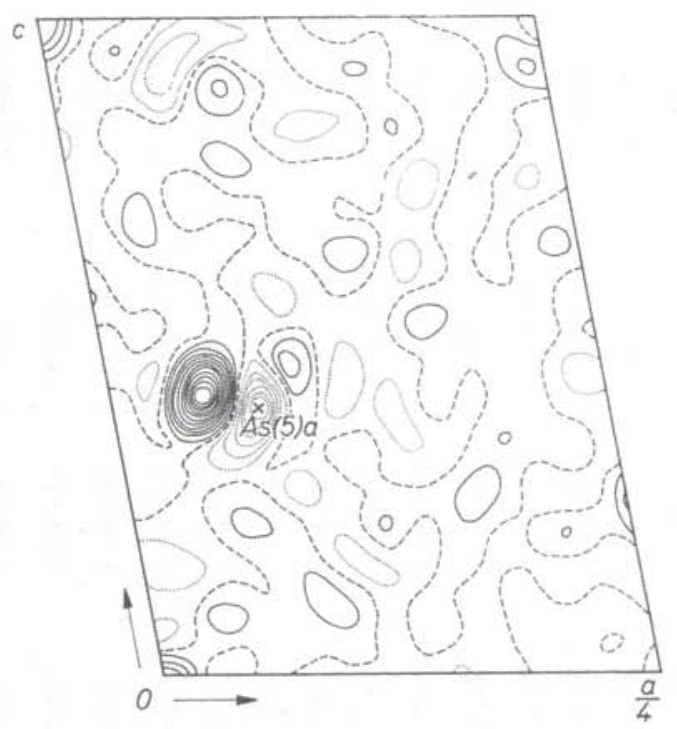

Fig. 1. A section of the three dimensional difference FouRrer map through the As(5) atom. Contours are at intervals of $4 e \cdot \mathrm{A}^{-3}$. The zero contour is shown as a dotted line and negative contours as broken lines

To clarify this point, a three-dimensional difference Fourier including 3477 diffraction data was calculated with the O.S. Mrus' programme for the Mercury computer at the calculating center of Oxford University. A part of the section through the As(5) atom is shown in Fig.1, in which a negative region at the postulated As(5) position and the peak near it is clearly observed, suggesting a statistical distribution of the $\mathrm{As}(5)$ atom between the two positions.

Three-dimensional least-squares refinements using equal weights for all reflections and assuming the statisticall distribution of the $\mathrm{As}(5)$ atom were then carried out with the programme written by C. T. PrewitT for the I.B.M. 7090 computer. Anisotropic temperature 


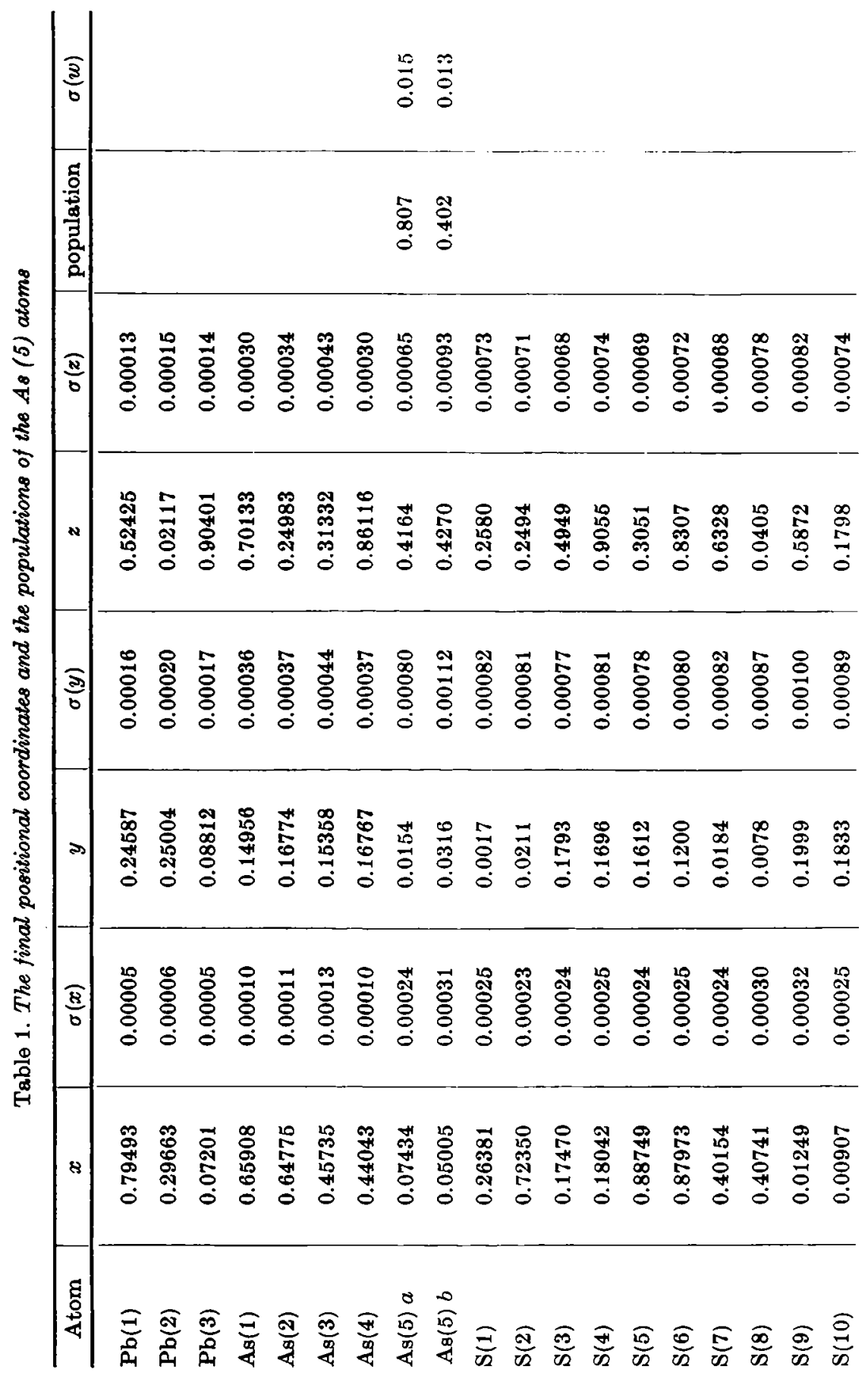


The crystal structure of rathite-I

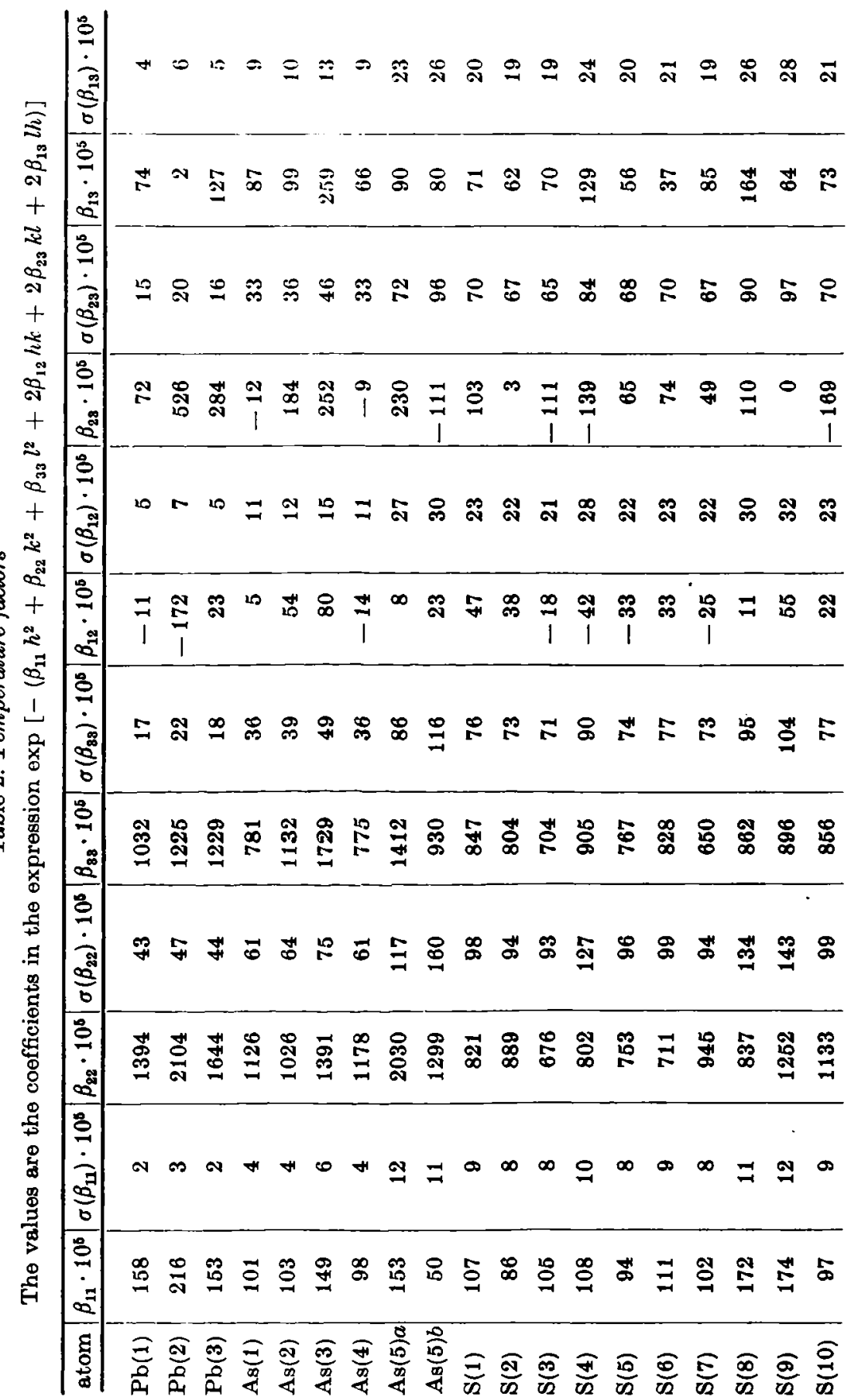


Table 3. The calculated and the observed structure amplitudes

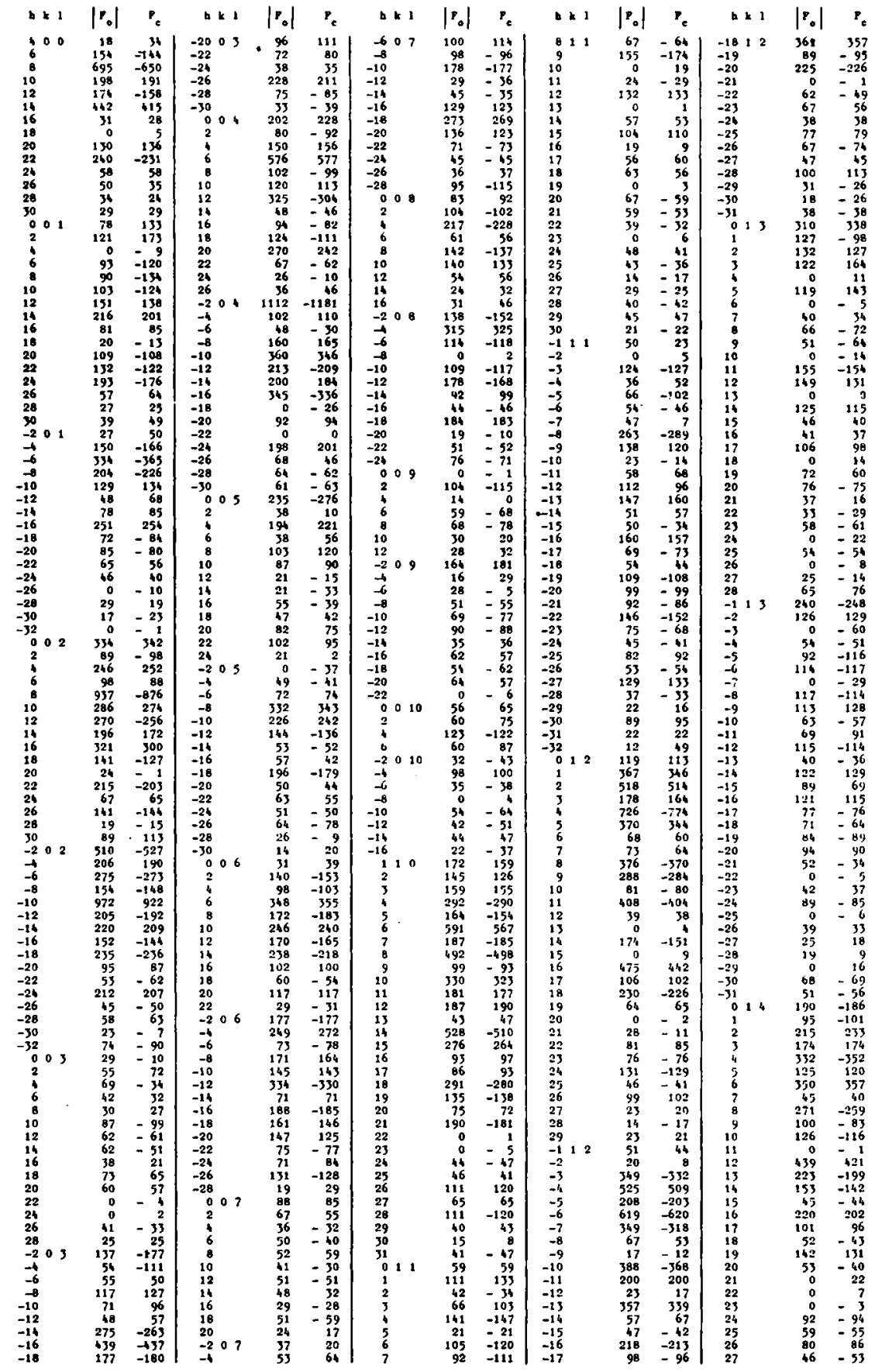


Table 3. (Continued)

\begin{tabular}{|c|c|c|c|c|c|c|c|c|c|c|c|c|}
\hline$b 11$ & $\left|P_{0}\right|$ & $p_{c}$ & $b 1$ & $\left|p_{0}\right|$ & $\mathbf{P}_{\mathrm{c}}$ & $b<1$ & $\left|P_{0}\right|$ & $\mathbf{F}_{\mathrm{c}}$ & $b \geq 1$ & $p_{0}$ & $p_{c}$ & $b * 1$ \\
\hline$-1: 1$ & 202 & -208 & 1516 & 74 & -64 & -118 & 26 & 35 & 1820 & th & 50 & 2622 \\
\hline-2 & $3 t$ & 25 & & 122 & 116 & & 117 & 121 & & 98 & -104 & 27 \\
\hline $\begin{array}{l}-3 \\
-6\end{array}$ & $\begin{array}{l}10 ! \\
103\end{array}$ & $\begin{array}{r}-89 \\
100\end{array}$ & 17 & $\begin{array}{l}56 \\
{ }_{78}\end{array}$ & $\begin{array}{l}=53 \\
-32\end{array}$ & $\begin{array}{l}-3 \\
-4\end{array}$ & 113 & $\begin{array}{r}117 \\
-20\end{array}$ & $\begin{array}{l}20 \\
21\end{array}$ & $\begin{array}{r}237 \\
55\end{array}$ & $\begin{array}{r}-235 \\
61\end{array}$ & $\begin{array}{l}28 \\
29\end{array}$ \\
\hline $\begin{array}{l}-4 \\
-5\end{array}$ & $\begin{array}{r}103 \\
i 7\end{array}$ & $\begin{array}{r}100 \\
-67\end{array}$ & $\begin{array}{l}18 \\
19\end{array}$ & $\begin{array}{l}38 \\
25\end{array}$ & $\begin{array}{l}-32 \\
-24\end{array}$ & $\begin{array}{l}-4 \\
-5\end{array}$ & 64 & $\begin{array}{r}-20 \\
62\end{array}$ & $\begin{array}{l}21 \\
22\end{array}$ & $\begin{array}{r}55 \\
158\end{array}$ & $\begin{array}{l}61 \\
163\end{array}$ & $\begin{array}{rl}29 & 22\end{array}$ \\
\hline-6 & $3+1$ & -361 & 20 & 27 & -26 & -6 & 76 & -73 & 23 & 15 & -37 & -2 \\
\hline-7 & 174 & 173 & 21 & 36 & 30 & -7 & 0 & 9 & 24 & 91 & -101 & -3 \\
\hline $\begin{array}{l}-8 \\
-9\end{array}$ & 400 & 612 & 22 & & 98 & -8 & 108 & 203 & 25 & 0 & -19 & -4 \\
\hline $\begin{array}{l}-9 \\
10\end{array}$ & $\begin{array}{l}190 \\
350\end{array}$ & $\begin{array}{r}185 \\
-240\end{array}$ & $\begin{array}{lll}-1 & 1 & 6\end{array}$ & 164 & -155 & -9 & 144 & $-14 I$ & 26 & 59 & 60 & -5 \\
\hline $\begin{array}{l}10 \\
11\end{array}$ & 258 & $\begin{array}{r}-240 \\
81\end{array}$ & $\begin{array}{l}-2 \\
-3\end{array}$ & 76 & -82 & -10 & 157 & -159 & 27 & if & 49 & -6 \\
\hline 12 & 192 & 187 & -7 & 0 & $\begin{array}{l}-19 \\
-16\end{array}$ & $\begin{array}{l}-11 \\
-12\end{array}$ & $\begin{array}{r}61 \\
102\end{array}$ & $\begin{array}{r}-5 B \\
-105\end{array}$ & ${ }_{29}^{28}$ & $\begin{array}{l}20 \\
16\end{array}$ & $\begin{array}{r}13 \\
-19\end{array}$ & $\begin{array}{l}-7 \\
-8\end{array}$ \\
\hline & $13:$ & -128 & -5 & $1 ; 1$ & $\begin{array}{r}170 \\
173\end{array}$ & $\begin{array}{l}-12 \\
-13\end{array}$ & 0 & $\begin{array}{r}103 \\
-9\end{array}$ & 30 & i5 & & $\overrightarrow{-9}$ \\
\hline & $19 i$ & 183 & -6 & 266 & -265 & -14 & 72 & -77 & 31 & is & & -10 \\
\hline & 70 & -70 & -7 & 160 & 137 & -15 & 39 & 32 & 021 & 3 & 59 & -11 \\
\hline 16 & 266 & -242 & -8 & 317 & 330 & -16 & 93 & -87 & 1 & 24 & 5 & -12 \\
\hline-17 & 150 & -146 & -9 & 302 & 89 & -17 & 30 & 18 & 2 & 37 & & -13 \\
\hline 10 & 95 & 98 & -10 & 144 & -141 & -18 & 89 & 91 & 3 & 142 & -161 & -24 \\
\hline 19 & 0 & -12 & $-1 t$ & 104 & -106 & -19 & 21 & 22 & 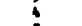 & 22 & 21 & -15 \\
\hline-20 & 170 & -178 & -12 & 127 & 125 & -20 & 95 & -107 & 5 & 163 & -173 & -16 \\
\hline $\begin{array}{l}-21 \\
-22\end{array}$ & 96 & $\begin{array}{l}61 \\
91\end{array}$ & $\begin{array}{l}-13 \\
-16\end{array}$ & 73 & $\begin{array}{l}=79 \\
=39\end{array}$ & $\begin{array}{l}-21 \\
-22\end{array}$ & 45 & -47 & 6 & 32 & -1 & $\begin{array}{l}-17 \\
-18\end{array}$ \\
\hline-23 & 80 & $\begin{array}{l}91 \\
82\end{array}$ & $\begin{array}{l}-14 \\
-15\end{array}$ & $\begin{array}{l}12 \\
112\end{array}$ & $\begin{array}{l}-39 \\
-100\end{array}$ & $\begin{array}{l}-22 \\
-23\end{array}$ & 24 & - 11 & 8 & 71 & -30 & $\begin{array}{l}-18 \\
-19\end{array}$ \\
\hline-24 & 0 & & -16 & 161 & -162 & -24 & 33 & -11 & 9 & 129 & 133 & -20 \\
\hline-25 & 0 & -2 & -17 & 114 & 99 & -25 & 21 & & 10 & 0 & -11 & -21 \\
\hline $\begin{array}{l}-26 \\
-27\end{array}$ & 47 & $\begin{array}{r}-50 \\
-53\end{array}$ & $\begin{array}{l}-18 \\
-19\end{array}$ & 90 & 91 & 019 & 25 & -23 & 11 & 96 & 105 & -22 \\
\hline 227 & $\begin{array}{l}61 \\
52\end{array}$ & $\begin{array}{r}-53 \\
-58\end{array}$ & $\begin{array}{l}-19 \\
-20\end{array}$ & $\begin{array}{r}89 \\
128\end{array}$ & 89 & $\frac{1}{2}$ & 54 & 54 & 12 & $6 ?$ & -71 & -23 \\
\hline $\begin{array}{l}-28 \\
-29\end{array}$ & $\begin{array}{l}52 \\
55\end{array}$ & $\begin{array}{r}58 \\
-46\end{array}$ & $\begin{array}{l}-20 \\
-21\end{array}$ & $\begin{array}{r}128 \\
52\end{array}$ & $\begin{array}{r}-126 \\
50\end{array}$ & $\frac{2}{3}$ & 0 & $\begin{array}{l}24 \\
15\end{array}$ & 13 & 55 & 72 & $\begin{array}{l}-21 \\
-25\end{array}$ \\
\hline $\begin{array}{l}-29 \\
-30\end{array}$ & 67 & $=77$ & $\begin{array}{l}-21 \\
-22\end{array}$ & $\begin{array}{l}32 \\
117\end{array}$ & 121 & 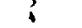 & 0 & 3 & 15 & 33 & ${ }_{8}^{37}$ & $\begin{array}{l}-25 \\
-26\end{array}$ \\
\hline-31 & 20 & $\begin{array}{l}=15 \\
=-62\end{array}$ & -23 & 0 & 7 & 5 & 46 & -47 & 16 & 27 & 28 & $\begin{array}{l}-27 \\
-27\end{array}$ \\
\hline & $\begin{array}{l}64 \\
35\end{array}$ & $\begin{array}{l}-62 \\
-45\end{array}$ & $\begin{array}{l}-24 \\
-25\end{array}$ & $\begin{array}{l}49 \\
56\end{array}$ & $\begin{array}{r}38 \\
-19\end{array}$ & $\begin{array}{l}6 \\
7\end{array}$ & $\begin{array}{r}42 \\
0\end{array}$ & $\begin{array}{l}=34 \\
-11\end{array}$ & $\begin{array}{l}17 \\
18\end{array}$ & $\begin{array}{r}176 \\
45\end{array}$ & $\begin{array}{r}-169 \\
37\end{array}$ & $\begin{array}{l}-20 \\
-29\end{array}$ \\
\hline 2 & 111 & 112 & -26 & 10 & io & 8 & 26 & 22 & 19 & 190 & -178 & -30 \\
\hline 3 & st & -4 & $\begin{array}{l}-27 \\
-28\end{array}$ & $\begin{array}{l}54 \\
27\end{array}$ & -31 & 10 & $\begin{array}{r}0 \\
27\end{array}$ & $\begin{array}{r}1 \\
22\end{array}$ & $\begin{array}{l}20 \\
21\end{array}$ & $\begin{array}{l}37 \\
36\end{array}$ & $\begin{array}{l}-32 \\
-27\end{array}$ & $\begin{array}{r}-31 \\
0\end{array}$ \\
\hline 3 & 0 & -17 & -29, & $\begin{array}{l}21 \\
56\end{array}$ & $\begin{array}{r}-20 \\
-52\end{array}$ & 11 & 0 & -37 & 22 & 0 & 8 & i \\
\hline$\frac{6}{7}$ & 131 & $\begin{array}{r}-11 \\
145\end{array}$ & & $\begin{array}{r}30 \\
103\end{array}$ & $\begin{array}{l}-32 \\
-118\end{array}$ & -119 & 0 & -38 & 24 & 22 & 9 & 3 \\
\hline g & 5 & ${ }_{54}^{17}$ & 2 & $\begin{array}{l}44 \\
76\end{array}$ & $\begin{array}{l}-49 \\
-86\end{array}$ & $\begin{array}{l}-2 \\
-3\end{array}$ & 30 & 32 & $\begin{array}{l}25 \\
26\end{array}$ & 71 & $\begin{array}{r}72 \\
-\quad 3\end{array}$ & 5 \\
\hline 10 & 69 & -66 & ? & $\begin{array}{r}70 \\
0\end{array}$ & $\begin{array}{r}-90 \\
6\end{array}$ & $\rightarrow$ & 83 & 87 & $\begin{array}{l}20 \\
27\end{array}$ & $\begin{array}{l}22 \\
85\end{array}$ & 87 & 6 \\
\hline 11 & 17 & -4 & 5 & 67 & -63 & -5 & 30 & -25 & 28 & 0 & -3 & 7 \\
\hline $\begin{array}{l}12 \\
13\end{array}$ & $\begin{array}{l}35 \\
61\end{array}$ & $\begin{array}{l}-34 \\
-58\end{array}$ & 7 & $\begin{array}{r}109 \\
0\end{array}$ & $\begin{array}{r}95 \\
0\end{array}$ & $\begin{array}{l}-6 \\
-7\end{array}$ & ${ }^{68}$ & $=74$ & 39 & 39 & 25 & : \\
\hline 14 & 0 & 14 & 8 & 0 & 17 & - & 47 & -50 & $\begin{array}{rll}30 & 21\end{array}$ & 230 & 1 & 10 \\
\hline 15 & 0 & -8 & 9 & 97 & 101 & -9 & 78 & -81 & -2 & 0 & & II \\
\hline $\begin{array}{l}16 \\
17\end{array}$ & 0 & $\begin{array}{r}-8 \\
-5\end{array}$ & 10 & $\begin{array}{c}86 \\
55\end{array}$ & $\begin{array}{r}-75 \\
52\end{array}$ & $\begin{array}{l}-10 \\
-11\end{array}$ & 29 & 26 & -3 & 178 & 204 & 12 \\
\hline 18 & 0 & 19 & 12 & 39 & -38 & -12 & $\begin{array}{r}23 \\
120\end{array}$ & $\begin{array}{r}18 \\
-125\end{array}$ & -5 & $\begin{array}{l}31 \\
55\end{array}$ & -3 & 16 \\
\hline 19 & $\pi$ & 69 & 13 & 28 & -21 & -13 & Ba & 86 & -6 & 34 & & 15 \\
\hline 20 & 32 & -27 & 14 & 43 & $-4 i$ & -14 & 41 & -47 & -7 & 58 & -64 & 16 \\
\hline 21 & 0 & 33 & is & 59 & -58 & -15 & $\because$ & -1 & -8 & 19 & $?$ & 17 \\
\hline 22 & 44 & -38 & 16 & 70 & $\begin{array}{l}-70 \\
-7\end{array}$ & -16 & 39 & 46 & -9 & 218 & -231 & 18 \\
\hline $\begin{array}{l}23 \\
24\end{array}$ & $\begin{array}{l}0 \\
0\end{array}$ & $\begin{array}{r}-2 \\
-7\end{array}$ & $\begin{array}{l}17 \\
18\end{array}$ & $\begin{array}{l}29 \\
37\end{array}$ & -24 & $\begin{array}{l}-17 \\
-10\end{array}$ & 70 & 75 & $\begin{array}{l}-10 \\
-11\end{array}$ & $\begin{array}{r}94 \\
266\end{array}$ & $\begin{array}{r}91 \\
-272\end{array}$ & $\begin{array}{l}19 \\
20\end{array}$ \\
\hline 25 & 0 & 4 & 19 & 22 & -16 & -19 & 0 & 10 & $\begin{array}{l}-11 \\
-12\end{array}$ & 120 & 1 & 21 \\
\hline-1 & 0 & -37 & & & 30 & -20 & 42 & 41 & -13 & $6 ?$ & -66 & 22 \\
\hline-2 & $\begin{array}{r}99 \\
125\end{array}$ & -108 & $\begin{array}{ccc}-1 & 1 & 7\end{array}$ & 80 & 72 & -21 & 26 & -34 & -14 & 39 & 32 & 23 \\
\hline-3 & $\begin{array}{l}125 \\
196\end{array}$ & $\begin{array}{r}96 \\
197\end{array}$ & -2 & 166 & $\begin{array}{r}-186 \\
-162\end{array}$ & -12 & 11 & -9 & -15 & 106 & 117 & 26 \\
\hline $\begin{array}{l}-4 \\
-5\end{array}$ & $\begin{array}{l}190^{\circ} \\
199\end{array}$ & 194 & -3 & $\begin{array}{l}358 \\
128 \\
128\end{array}$ & $\begin{array}{r}162 \\
-131\end{array}$ & o. 10 & $\begin{array}{l}88 \\
60\end{array}$ & $\begin{array}{r}-107 \\
64\end{array}$ & $\begin{array}{l}-16 \\
-17\end{array}$ & $\begin{array}{r}128 \\
58\end{array}$ & $\begin{array}{r}-133 \\
61\end{array}$ & 25 \\
\hline $\begin{array}{l}-3 \\
-6 \\
-7\end{array}$ & 67 & 52 & -5 & 0 & $=7$ & $\frac{1}{2}$ & 55 & 68 & $\begin{array}{l}-17 \\
-18\end{array}$ & 78 & -78 & $\begin{array}{l}20 \\
27\end{array}$ \\
\hline $\begin{array}{l}-7 \\
-8\end{array}$ & $\begin{array}{r}86 \\
0\end{array}$ & $\begin{array}{r}106 \\
12\end{array}$ & $\begin{array}{l}-6 \\
-7\end{array}$ & $\begin{array}{r}0 \\
53\end{array}$ & 5 & 3 & 38 & $\begin{array}{l}-27 \\
=40\end{array}$ & $\begin{array}{l}-19 \\
-20\end{array}$ & 39 & $=47$ & ${ }_{-12}^{28}$ \\
\hline $\begin{array}{l}-0 \\
-9\end{array}$ & ys & -77 & $\begin{array}{l}-1 \\
-8\end{array}$ & 95 & 95 & 5 & to & $=41$ & -21 & 73 & & $\begin{array}{l}-1 \\
-2\end{array}$ \\
\hline-10 & 331 & 33 & -9 & 34 & 41 & 6 & 19 & -18 & -22 & 114 & -10 & -3 \\
\hline-11 & 177 & -161 & -10 & 44 & 34 & -1110 & 74 & 70 & -23 & 0 & & -4 \\
\hline-12 & 50 & 49 & -11 & 52 & -58 & -2 & 125 & 141 & -24 & 71 & , & -5 \\
\hline-1 & 00 & -3 & -12 & 0 & -26 & -3 & 38 & 37 & -25 & 69 & & -6 \\
\hline-14 & 108 & -108 & -13 & 71 & -74 & -4 & 0 & 10 & -26 & 0 & & -7 \\
\hline $\begin{array}{l}-15 \\
-16\end{array}$ & 143 & -157 & $\begin{array}{l}-14 \\
-15\end{array}$ & 137 & 131 & -5 & 19 & $\begin{array}{r}-22 \\
30\end{array}$ & $\begin{array}{l}-27 \\
-28\end{array}$ & 67 & 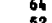 & $\rightarrow$ \\
\hline-1 & 0 & $\begin{array}{l}=15 \\
-19\end{array}$ & $\begin{array}{l}-15 \\
-16\end{array}$ & 63 & $\begin{array}{r}30 \\
-56\end{array}$ & $\begin{array}{l}-6 \\
-7\end{array}$ & 75 & $\begin{array}{r}30 \\
-80\end{array}$ & $\begin{array}{l}-28 \\
-29\end{array}$ & $\begin{array}{l}50 \\
22\end{array}$ & 52 & $\begin{array}{r}-9 \\
-10\end{array}$ \\
\hline-1 & 117 & -105 & -17 & 28 & -1 & -8 & 87 & & -30 & 13 & & -11 \\
\hline-1 & 67 & 73 & -18 & 110 & -98 & -9 & 31 & $-3 i$ & $-\mathbf{3 1}$ & 8 & & -12 \\
\hline-2 & 0 & -19 & -19 & 38 & 37 & -10 & 136 & -167 & 0 & 365 & -3 & -13 \\
\hline-2 & 101 & 96 & -20 & 83 & $7 i$ & -11 & 0 & 5 & 1 & isi & -1 & -16 \\
\hline-2 & 43 & 48 & -21 & 41 & 31 & -12 & 72 & 88 & 2 & 73 & 75 & -15 \\
\hline-2 & $\begin{array}{r}71 \\
146\end{array}$ & 68 & -22 & 47 & -41 & -13 & 63 & 0 & 3 & 312 & -2 & -16 \\
\hline-25 & $\begin{array}{r}146 \\
74\end{array}$ & ${ }_{71}^{141}$ & $\begin{array}{l}-23 \\
-24\end{array}$ & 34 & 20 & $\begin{array}{l}-14 \\
-15\end{array}$ & $\stackrel{0}{12}$ & -9 & 1 & 114 & & -17 \\
\hline-26 & 56 & $\begin{array}{l}77 \\
56\end{array}$ & $\begin{array}{l}-21 \\
-25\end{array}$ & 36 & $=99$ & $\begin{array}{l}-15 \\
-16\end{array}$ & 14 & 36 & 6 & $\begin{array}{r}0 \\
102\end{array}$ & & $\begin{array}{l}-18 \\
-19\end{array}$ \\
\hline-27 & $5 y$ & -61 & -26 & 31 & 29 & -17 & 16 & 13 & 7 & 75 & & -20 \\
\hline-28 & 0 & 18 & -27 & 0 & -7 & 02 & 679 & -468 & 8 & 231 & 2 & -21 \\
\hline-29 & 101 & -112 & 018 & 167 & $-17 i$ & 1 & 0 & -1 & 9 & 46 & - & -22 \\
\hline-30 & 42 & 47 & 1 & 116 & -112 & $\frac{2}{2}$ & 447 & 462 & 10 & 50 & & -23 \\
\hline $\begin{array}{l}016 \\
1\end{array}$ & $\begin{array}{l}302 \\
136\end{array}$ & $\begin{array}{r}-309 \\
-30\end{array}$ & & 146 & 169 & 3 & 326 & -330 & 11 & 255 & 2 & -24 \\
\hline $\begin{array}{l}1 \\
2\end{array}$ & $\begin{array}{l}136 \\
382\end{array}$ & $\begin{array}{r}-132 \\
12\end{array}$ & 3 & 92 & -90 & 4 & 246 & $\begin{array}{l}-231 \\
-560\end{array}$ & 12 & 216 & 2 & $\begin{array}{l}-25 \\
-26\end{array}$ \\
\hline 3 & $\begin{array}{l}382 \\
162\end{array}$ & $\begin{array}{r}\$ 12 \\
-167\end{array}$ & 5 & $\begin{array}{l}92 \\
37\end{array}$ & $=98$ & $\begin{array}{l}5 \\
6\end{array}$ & 563 & $\begin{array}{l}-560 \\
-520\end{array}$ & $\begin{array}{l}13 \\
14\end{array}$ & 83 & - & $\begin{array}{l}-26 \\
-27\end{array}$ \\
\hline 4 & 124 & -125 & 6 & 218 & 11 & 7 & 241 & -211 & 15 & 45 & & -28 \\
\hline 5 & 32 & 24 & 7 & 49 & 43 & B & 193 & 2 & 16 & 0 & & -29 \\
\hline 6 & 149 & 151 & e & 80 & 72 & 9 & 258 & 269 & 17 & 0 & - & -30 \\
\hline 7 & 163 & 161 & 9 & $\stackrel{0}{0}$ & 1 & 10 & 326 & -322 & 18 & 141 & 140 & -31 \\
\hline 8 & 72 & 73 & 10 & 136 & -147 & 11 & 151 & 158 & 19 & 121 & -116 & 02 \\
\hline$y_{10}^{y}$ & 192 & 189 & 11 & 95 & 87 & 12 & 395 & $\begin{array}{r}404 \\
50\end{array}$ & 20 & .46 & -43 & 1 \\
\hline $\begin{array}{l}10 \\
11\end{array}$ & $\begin{array}{l}87 \\
30\end{array}$ & $\begin{array}{l}-78 \\
-30\end{array}$ & $\begin{array}{l}12 \\
13\end{array}$ & $\begin{array}{r}87 \\
0\end{array}$ & $\begin{array}{r}90 \\
-12\end{array}$ & ${ }_{14}^{13}$ & $\begin{array}{r}42 \\
121\end{array}$ & $\begin{array}{r}50 \\
-121\end{array}$ & $\begin{array}{l}21 \\
22\end{array}$ & $\begin{array}{l}126 \\
109\end{array}$ & $\begin{array}{r}-1 \\
1\end{array}$ & $\frac{2}{3}$ \\
\hline 12 & 56 & 52 & 16 & 98 & -92 & $\begin{array}{l}14 \\
15\end{array}$ & 95 & 94 & 23 & 0 & -8 & 4 \\
\hline 13 & 0 & 2 & 15 & 21 & 25 & 16 & 36 & 19 & 24 & 69 & -60 & 5 \\
\hline & 246 & & & +2 & 48 & 17 & 166 & 77 & 25 & 106 & 15 & 6 \\
\hline
\end{tabular}


Table 3. (Conlinued)

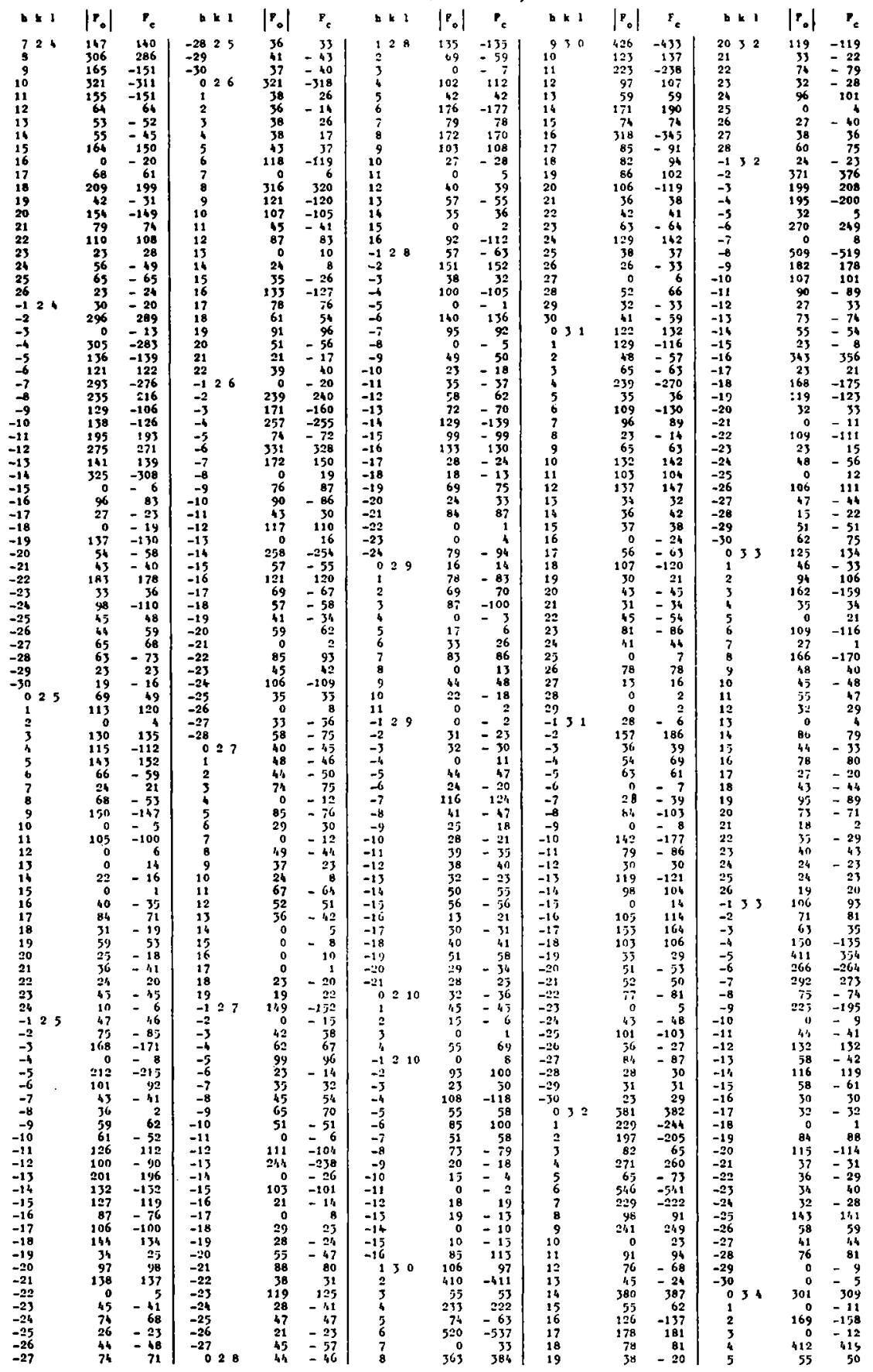


Table 3. (Continued)

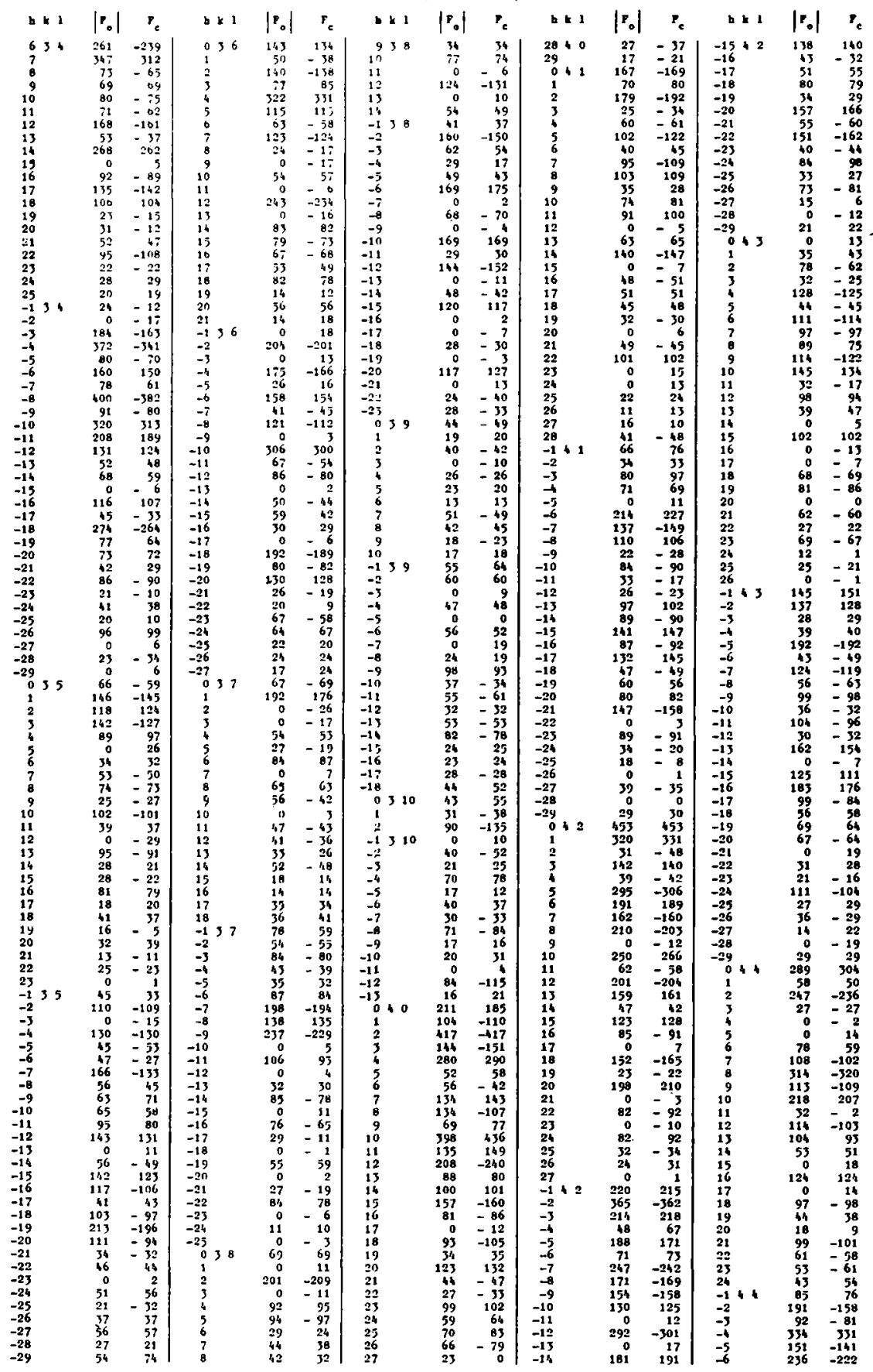


Table 3. (Continued)

\begin{tabular}{|c|c|c|c|c|c|c|c|}
\hline 11 & | $\mathbf{P}_{0} \mid$ & $\mathbf{P}_{\mathrm{c}}$ & $b \leq 1$ & $\left|p_{0}\right|$ & $F_{c}$ & b $<1$ & $\left|r_{0}\right|$ \\
\hline $\begin{array}{l}-7 \\
-8\end{array}$ & $\begin{array}{r}22 \\
0\end{array}$ & $\begin{array}{r}20 \\
-\quad 18\end{array}$ & $\begin{array}{l}-10 \\
-11\end{array}$ & $\begin{array}{l}131 \\
119\end{array}$ & $\begin{array}{l}117 \\
108\end{array}$ & $\begin{array}{l}-2 \\
-3\end{array}$ & $\begin{array}{l}78 \\
80\end{array}$ \\
\hline & 340 & $\begin{array}{r}-18 \\
122\end{array}$ & & ${ }_{74}^{19}$ & $\begin{array}{r}108 \\
-73\end{array}$ & & 64 \\
\hline-10 & 162 & 136 & -13 & 0 & & -5 & 66 \\
\hline-11 & 42 & 45 & -14 & 189 & 182 & -6 & 32 \\
\hline-12 & 265 & -206 & -15 & +7 & -10 & $-i$ & 39 \\
\hline-13 & 58 & 56 & -16 & 99 & - 99 & $\rightarrow$ & 26 \\
\hline-14 & 218 & $20 y$ & -17 & 107 & -100 & -9 & 18 \\
\hline-15 & 59 & 52 & -18 & 0 & 16 & -10 & 65 \\
\hline $\begin{array}{l}-16 \\
-17\end{array}$ & 76 & -70 & -19 & 0 & -9 & -11 & 67 \\
\hline $\begin{array}{l}-17 \\
-18\end{array}$ & 96 & -74 & -20 & 0 & -2 & -12 & 33 \\
\hline-19 & th & $\begin{array}{r}51 \\
-37\end{array}$ & $\begin{array}{l}-21 \\
-221\end{array}$ & $\begin{array}{l}25 \\
63\end{array}$ & $\begin{array}{r}28 \\
-58 \\
-58\end{array}$ & $\begin{array}{l}-15 \\
-16\end{array}$ & 35 \\
\hline-20 & 46 & -36 & -23 & 74 & -38 & $\begin{array}{l}-18 \\
-15\end{array}$ & 11 \\
\hline-21 & 0 & -6 & -24 & 63 & 71 & -16 & 21 \\
\hline-22 & 115 & -116 & -23 & 32 & 35 & -17 & 59 \\
\hline-23 & 0 & 1 & -26 & 56 & $-8 i$ & 150 & 117 \\
\hline $\begin{array}{l}-24 \\
-25\end{array}$ & 26 & 28 & $0+7$ & 0 & -7 & 2 & 140 \\
\hline $\begin{array}{l}-25 \\
-26\end{array}$ & $\begin{array}{l}51 \\
45\end{array}$ & $\begin{array}{r}48 \\
-50\end{array}$ & $\begin{array}{l}1 \\
2\end{array}$ & 0 & 30 & 3 & 35 \\
\hline $\begin{array}{l}-26 \\
-27\end{array}$ & 11 & 15 & 3 & 37 & $-3 n$ & 3 & 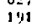 \\
\hline-28 & 40 & 48 & 4 & 104 & 96 & 6 & 105 \\
\hline $0+5$ & $22: 2$ & 208 & 5 & 131 & 123 & 7 & 139 \\
\hline 1 & 34 & -19 & 6 & 38 & -30 & 8 & 86 \\
\hline $\begin{array}{l}2 \\
3\end{array}$ & 77 & 70 & ? & $\begin{array}{c}101 \\
86\end{array}$ & $\begin{array}{r}103 \\
-87\end{array}$ & 10 & $\begin{array}{l}49 \\
11\end{array}$ \\
\hline & BI & -69 & 9 & 0 & -3 & 11 & 55 \\
\hline 5 & 36 & 33 & 16 & 36 & -32 & 12 & 119 \\
\hline 6 & 124 & -105 & 11 & 27 & 25 & 13 & 91 \\
\hline 7 & B3 & -73 & 12 & 31 & 28 & 14 & 35 \\
\hline a & 102 & -89 & 13 & 52 & -49 & 15 & 29 \\
\hline 9 & 47 & 28 & 14 & 26 & 22 & 16 & 193 \\
\hline 10 & 33 & -31 & 15 & 19 & -12 & 17 & 0 \\
\hline 11 & 0 & + & 16 & 10 & 39 & 18 & 173 \\
\hline 12 & $10 \mathrm{H}$ & 98 & -147 & & -7 & 19 & 53 \\
\hline 13 & 46 & -44 & -2 & 0 & -12 & 20 & 32 \\
\hline $\begin{array}{l}14 \\
15\end{array}$ & 77 & 74 & -3 & 103 & -106 & 21 & $2^{n}$ \\
\hline $\begin{array}{l}15 \\
16\end{array}$ & 20 & $\begin{array}{r}24 \\
-\quad 9\end{array}$ & -5 & 34 & $\begin{array}{l}-39 \\
-29\end{array}$ & 23 & 26 \\
\hline 17 & 36 & 39 & -6 & 0 & -8 & 24 & 33 \\
\hline 18 & 20 & -17 & -7 & $14 \bar{B}$ & 140 & 25 & i) \\
\hline 19 & 0 & 10 & -8 & 39 & is & 26 & he \\
\hline 20 & 60 & -59 & -9 & 101 & & 27 & 27 \\
\hline 21 & 26 & & -10 & 81 & 69 & 051 & 3 \\
\hline$\frac{22}{-1}+5$ & 100 & 16 & -11 & 0 & 15 & $?$ & 186 \\
\hline $\begin{array}{ll}-1 & 3\end{array}$ & $\begin{array}{l}140 \\
137\end{array}$ & $\begin{array}{r}-129 \\
109\end{array}$ & $\begin{array}{l}-12 \\
-13\end{array}$ & 65 & $\begin{array}{l}15 \\
55\end{array}$ & $\frac{2}{3}$ & 43 \\
\hline-3 & 80 & -75 & -14 & 30 & $2 \bar{j}$ & 4 & 0 \\
\hline-4 & 45 & -66 & -15 & 67 & -52 & 5 & 11 \\
\hline-5 & $n$ & -6 & -16 & 33 & 26 & 6 & 37 \\
\hline-6 & 28 & 23 & -17 & 79 & -64 & $?$ & 240 \\
\hline $\begin{array}{l}-7 \\
-8\end{array}$ & 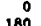 & -6 & -18 & 131 & -123 & 8 & 78 \\
\hline $\begin{array}{l}-8 \\
-9\end{array}$ & $\begin{array}{l}180 \\
144\end{array}$ & $\begin{array}{r}-162 \\
121\end{array}$ & $\begin{array}{l}-19 \\
-20\end{array}$ & 52 & $\begin{array}{r}55 \\
-40\end{array}$ & ${ }_{10}^{9}$ & 141 \\
\hline-10 & 90 & -81 & $\begin{array}{l}-2013 \\
-21\end{array}$ & $\begin{array}{r}77 \\
0\end{array}$ & -49 & it & 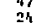 \\
\hline-11 & 58 & $5 y$ & -22 & 47 & 48 & 12 & 0 \\
\hline-12 & 63 & 40 & -25 & 0 & -11 & 13 & 84 \\
\hline-13 & 34 & -27 & & 17 & -25 & 14 & 0 \\
\hline $\begin{array}{l}-14 \\
-15\end{array}$ & 8 & -67 & 048 & 106 & 105 & 15 & 1.10 \\
\hline $\begin{array}{l}-15 \\
-16\end{array}$ & 207 & $\begin{array}{r}-185 \\
38\end{array}$ & 1 & 0 & 15 & 16 & 43 \\
\hline-17 & 136 & -120 & 3 & 50 & $\begin{array}{r}52 \\
-51\end{array}$ & $\begin{array}{l}17 \\
18\end{array}$ & 年 \\
\hline-18 & 57 & 59 & 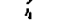 & 44 & -36 & i) & 8 \\
\hline-19 & 81 & -83 & 5 & 41 & 39) & 20 & n9 \\
\hline-20 & 56 & 63 & 6 & 107 & 117 & 21 & 66 \\
\hline-21 & 108 & -102 & $?$ & 39 & 33 & 22 & 22 \\
\hline $\begin{array}{l}-22 \\
-23 \\
-23\end{array}$ & 78 & -72 & 8 & $10 \%$ & -112 & 23 & 6 \\
\hline $\begin{array}{l}-23 \\
-26\end{array}$ & 112 & 118 & $?$ & 0 & -18 & 24 & 17 \\
\hline $\begin{array}{l}-26 \\
-25\end{array}$ & 74 & -14 & 10 & 59 & 28 & $\begin{array}{l}25 \\
25\end{array}$ & 17 \\
\hline-26 & 52 & 35 & 12 & 11 & $=-10$ & $\begin{array}{ll}-10 & 51\end{array}$ & $21 \frac{17}{3}$ \\
\hline-27 & 26 & $-3 i$ & 13 & $3 i$ & -34 & & 118 \\
\hline 046 & 86 & 92 & $-1+8$ & 58 & -50 & -5 & 19 \\
\hline 1 & $\because$ & -2 & & 175 & -182 & -4 & $2 y$ \\
\hline$\frac{2}{3}$ & 53 & 53 & -5 & $\begin{array}{l}31 \\
86\end{array}$ & -32 & -5 & 197 \\
\hline 3 & 132 & $\begin{array}{r}120 \\
-131\end{array}$ & -4 & 86 & & -6 & 0 \\
\hline 5 & 33 & -131 & $\begin{array}{l}-5 \\
-6\end{array}$ & 41 & -1 & -7 & 150 \\
\hline 6 & 163 & $1=0$ & -7 & 8 & $-1 n$ & 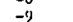 & 7 \\
\hline 7 & 80 & 73 & & 11,6 & 14 & -10 & 28 \\
\hline อ & 180 & $-17 i$ & $-1)$ & 67 & & -11 & 127 \\
\hline 9 & 40 & 34 & -10 & 47 & & -12 & 01 \\
\hline 10 & 132 & 123 & -11 & 0 & 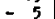 & -13 & $4 \overline{7}$ \\
\hline 11 & 103 & -88 & -12 & 73 & & -14 & 0 \\
\hline 12 & 27 & -18 & -13 & 39 & -47 & -15 & 85 \\
\hline 13 & 90 & -83 & $-1 / 1$ & 70 & & & 35 \\
\hline 14 & 31 & 35 & -15 & 0 & 10 & -17 & 48 \\
\hline 15 & 0 & I & -16 & 129 & -139 & -18 & 62 \\
\hline 16 & 65 & 60 & -17 & 24 & 22 & -19 & 63 \\
\hline 17 & 0 & 3 & -18 & 28 & 39 & -20 & 107 \\
\hline 16 & 125 & -139 & & 18 & 6 & -21 & 28 \\
\hline $\begin{array}{l}19 \\
20\end{array}$ & $\begin{array}{l}12 \\
31\end{array}$ & $\begin{array}{r}-5 \\
82\end{array}$ & $\begin{array}{l}-20 \\
-21\end{array}$ & $\begin{array}{l}16 \\
23\end{array}$ & $\begin{array}{r}-10 \\
-26\end{array}$ & $\begin{array}{l}-22 \\
-23\end{array}$ & $\begin{array}{l}17 \\
0\end{array}$ \\
\hline$-1 \leftrightarrow 6$ & 155 & $-13 y$ & 0 & 3 & -5 & -24 & 25 \\
\hline-2 & 264 & -250 & 1 & 0 & -2 & -25 & 37 \\
\hline-3 & 132 & -123 & 2 & 50 & 50 & -26 & 11 \\
\hline-4 & 157 & 155 & 3 & 0 & -4 & -27 & 0 \\
\hline-5 & 0 & $\begin{array}{r}20 \\
-125\end{array}$ & $t$ & 37 & 35 & 052 & 104 \\
\hline $\begin{array}{l}-6 \\
-7\end{array}$ & 136 & $\begin{array}{l}-125 \\
-30\end{array}$ & 6 & 20 & $\begin{array}{l}11 \\
31\end{array}$ & $\frac{1}{2}$ & $\begin{array}{r}00 \\
155\end{array}$ \\
\hline-8 & 87 & -64 & 7 & 25 & -28 & 3 & 175 \\
\hline-9 & 115 & 97 & -149 & 25 & -19 & 4 & 210 \\
\hline
\end{tabular}


Table 3. (Continued)

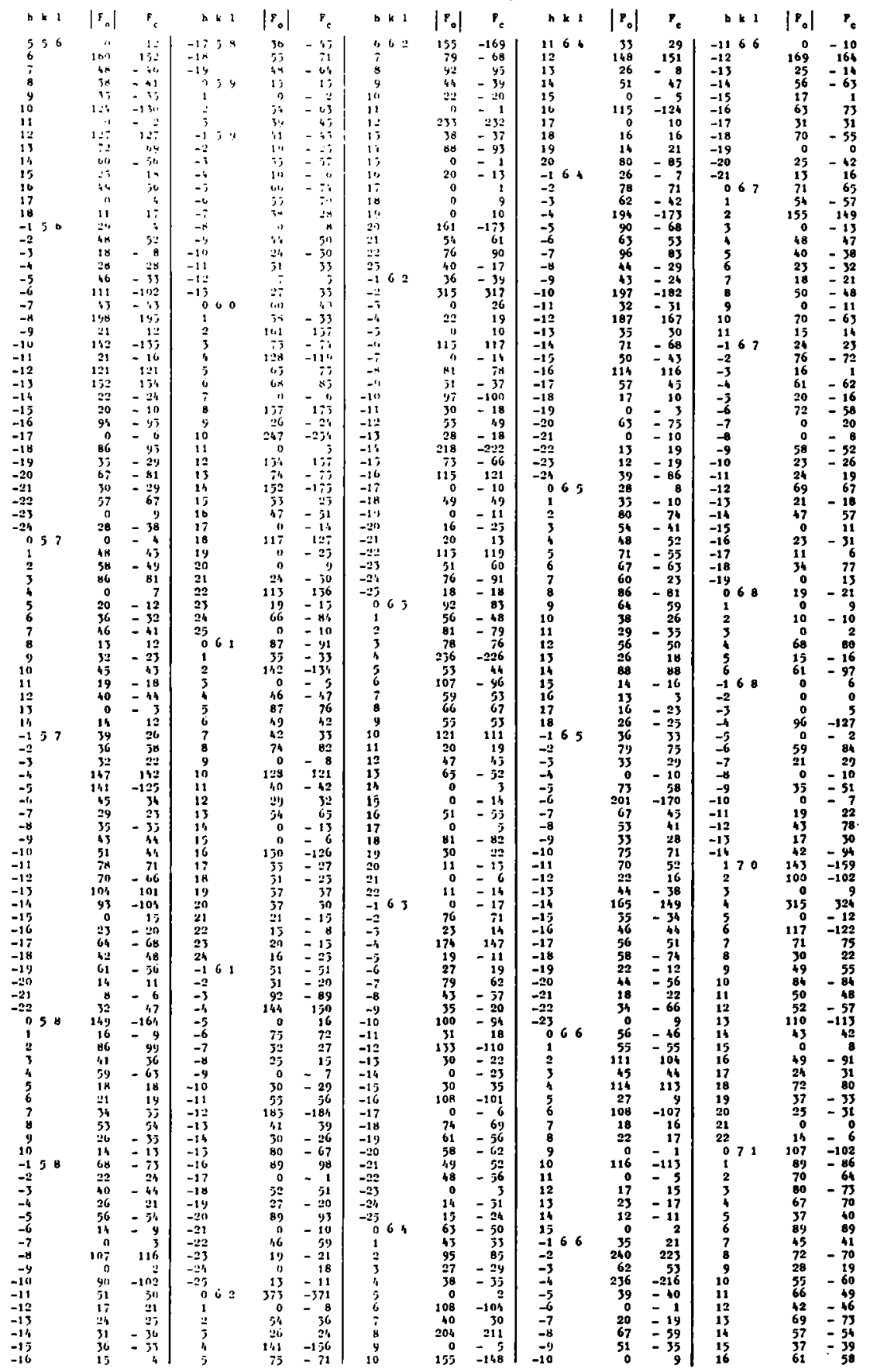


Table 3. (Continued)

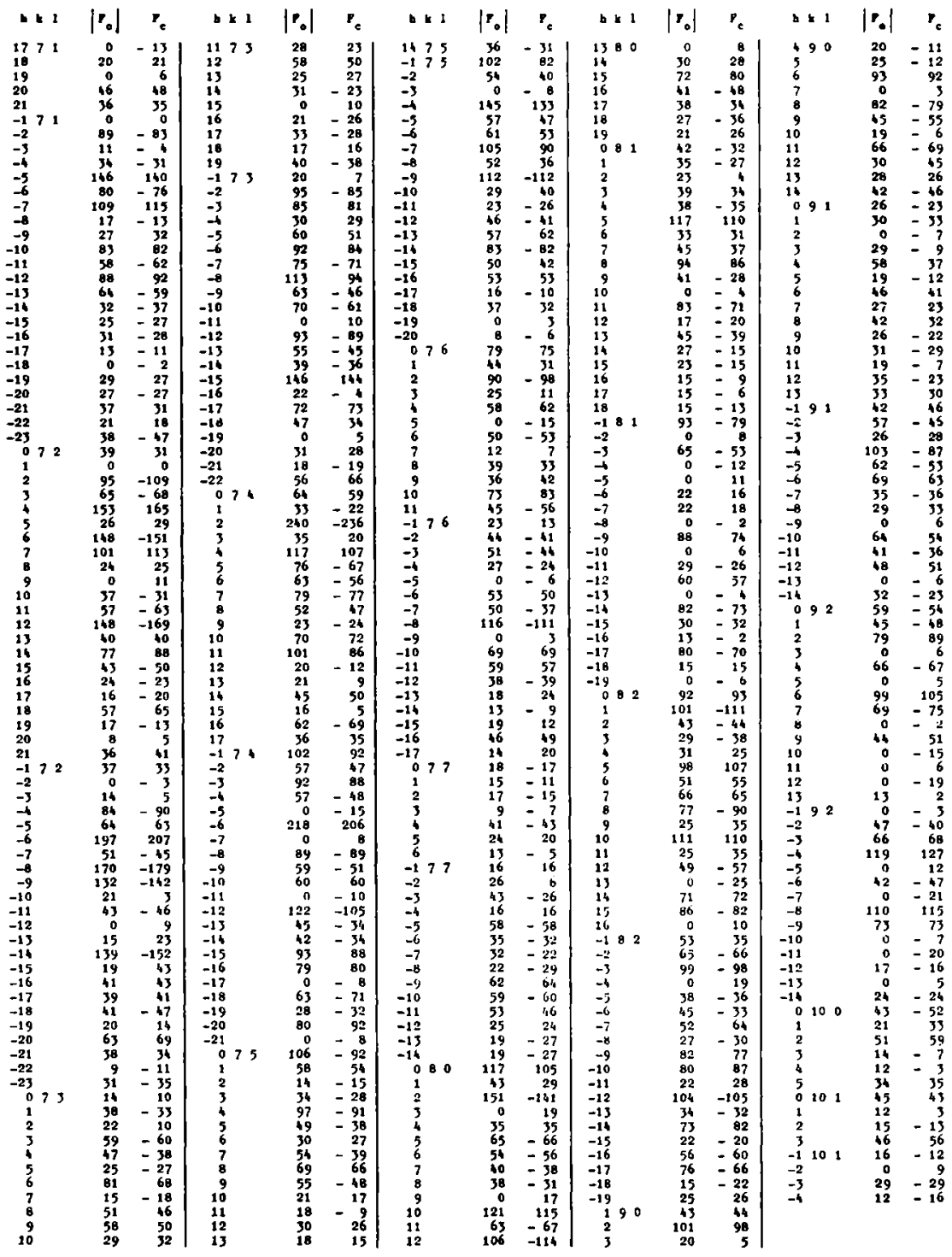

factors were introduced for the second cycle of the refinement. The atomic scattering factors given by B. Dawson, by A. J. Freemax and R. E. WAtson and by L. H. Thomas, K. Umeda and K. King (International Table, Vol. III, 1962) were used for $\mathrm{S}$, As and $\mathrm{Pb}$, respectively. Using this programme, the atomic coordinates, temperature factors, layer-scale factors and the population of the $\mathrm{As}(5)$ 
atom at the two positions were refined. After three cycles of refinement, the $R$ factor was reduced from the initial value of 0.23 to 0.102 for all 3477 reflections and 0.086 for the 3013 observed reflections. The experimentally determined relative layer-scale factors and the final values obtained by the least-squares refinement agree within $3 \%$, except for those reflections with $k$ larger than 7 which were obtained from the photographs around the $c$ axis. The experimental layer-scale factor for these reflections was underestimated owing to the insufficient integration for $K \alpha_{1}-K \alpha_{2}$ splitting in the higher Bragg-angle regions.

The final positional coordinates and the temperature factors are given in Table 1 and Table 2, respectively, with the standard deviations calculated by the least-squares programme. Since the dispersion effect was not taken into account, the actual temperature factors of the $\mathrm{Pb}$ atoms should be smaller than the values given in Table 2. The calculated and the observed structure amplitudes are given in Table 3. For the calculation of the structure amplitudes, the population of the $\mathrm{As}(5)$ atoms at the two positions were assumed to be 0.668 and 0.332 respectively.

The maximum and the average coordinate shifts in the last cycle of the refinement expressed as fractions of the standard deviations are 2.01 and 0.66 . Since we obtained a good convergence with the full-matrix least-squares programme, it is not expected that further refinement will cause significant changes in the atomic coordinates unless a new weighting scheme is employed.

\section{Deseription of the structure}

The atomic distances and the bond angles are given in Table 4. From the temperature factors the r.m.s. deviations of the atoms along the principal axes of the vibration ellipsoids were calculated and are given in Table 5 along with the direction cosines of two principal axes.

$\mathrm{Pb}(1)$ and $\mathrm{Pb}(2)$ are surrounded by nine $\mathrm{S}$ atoms in the manner shown in Fig.2. The coordination polyhedra around $\mathrm{Pb}(1)$ and $\mathrm{Pb}(2)$ are joined together by sharing the bases to form $\mathrm{PbS}_{6}$ strings along the $c$ axis direction. The strings are laterally combined by sharing triangular faces of the polyhedra and form $\mathrm{PbS}_{3}$ layers parallel to (100). $\mathrm{Pb}(3)$ has seven nearest-neighbouring $\mathrm{S}$ atoms. The mean $\mathrm{Pb}(3)-\mathrm{S}$ distance is somewhat shorter than the mean $\mathrm{Pb}(1), \mathrm{Pb}(2)-\mathrm{S}$ distances.

$\operatorname{As}(1), A s(2)$ and $A s(4)$ are each coordinated by three $S$ atoms forming trigonal pyramids with them, and these are joined into strings by sharing $\mathrm{S}$ atoms (Fig.3). The mean As $-\mathrm{S}$ distances agree 
F. Marumo and W. NowackI

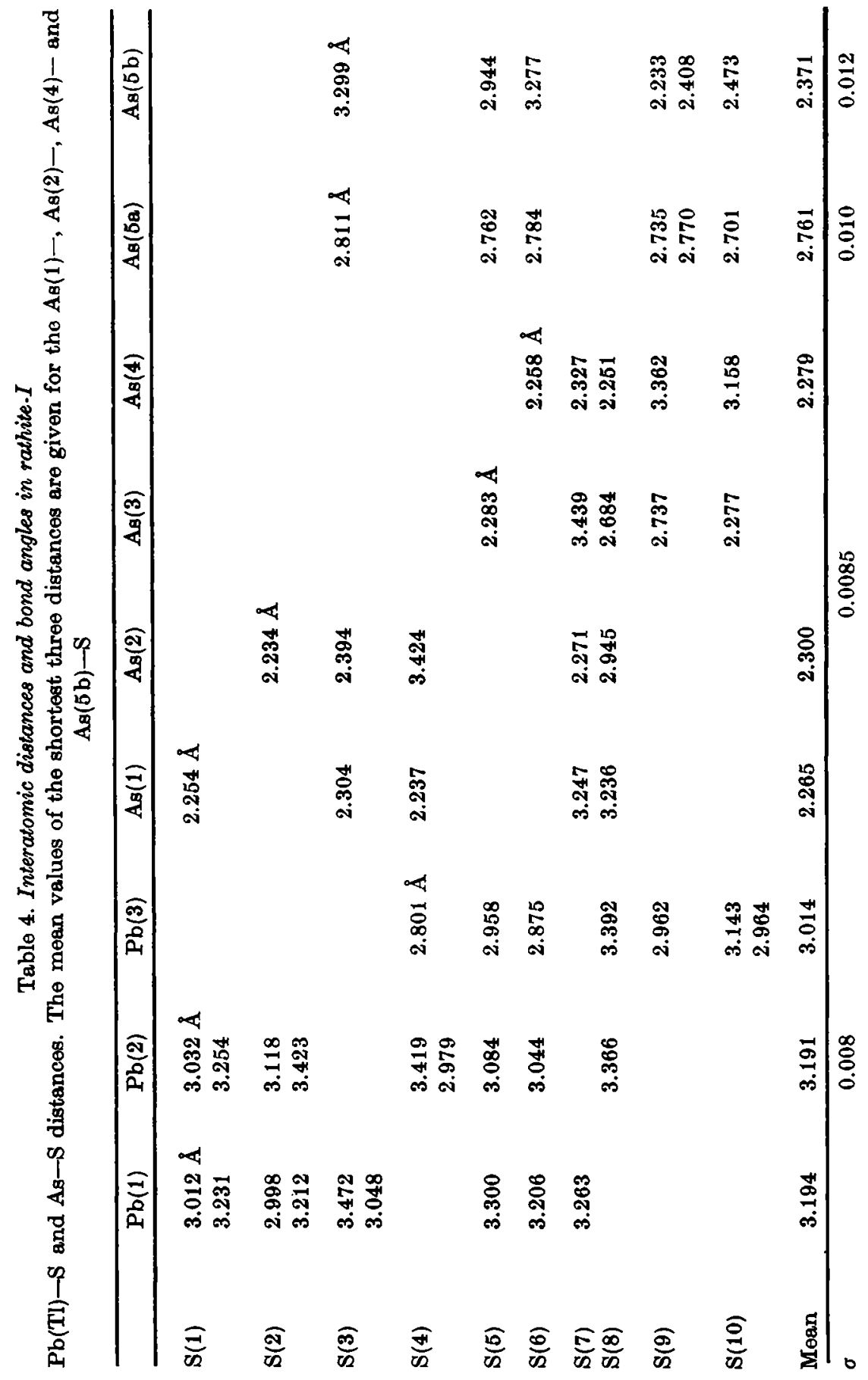


The crystal structure of rathite-I

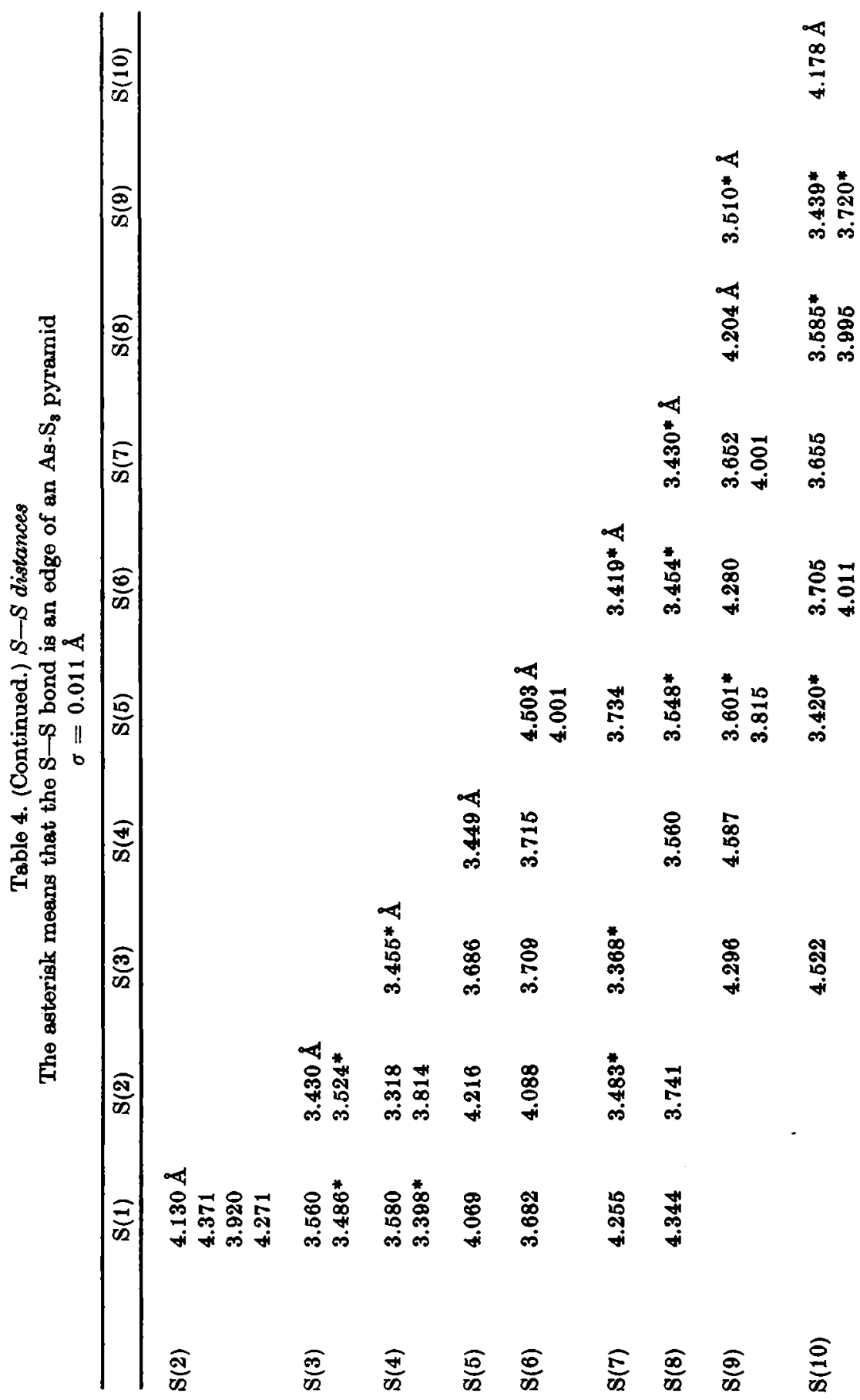


Table 4. (Continued)

\begin{tabular}{|c|c|c|c|}
\hline \multicolumn{4}{|c|}{ Bond angles } \\
\hline$S(1)-A s(1)-S(3)$ & $99.8^{\circ}$ & $S(5)-A s(3)-S(9)$ & $91.2^{\circ}$ \\
\hline$S(1)-A s(1)-S(4)$ & 98.3 & $S(9)-A s(3)-S(10)$ & 86.1 \\
\hline$S(3)-A s(1)-S(4)$ & 99.1 & $S(6)-A s(4)-S(7)$ & 96.4 \\
\hline$S(2)-A s(2)-S(3)$ & 99.1 & $S(6)-A s(4)-S(8)$ & 100.0 \\
\hline$S(2)-A s(2)-S(7)$ & 101.3 & $S(7)-A s(4)-S(8)$ & 97.0 \\
\hline$S(3)-A s(2)-S(7)$ & 92.4 & $\mathbf{S}(9)-\mathbf{A s}(5 b)-\mathbf{S}\left(9^{\prime}\right)$ & 98.2 \\
\hline$S(5)-A s(3)-S(8)$ & 90.8 & $\mathbf{S}(9)-\mathbf{A s}(5 b)-\mathrm{S}(10)$ & 99.3 \\
\hline$S(5)-A 8(3)-S(10)$ & 97.2 & $\mathbf{S}\left(9^{\prime}\right)-\mathbf{A s}(5 b)-\mathbf{S}(10)$ & 93.8 \\
\hline $\mathbf{S}(8)-\mathbf{A s}(\mathbf{3})-\mathbf{S}(10)$ & 92.2 & $\sigma \doteqdot 0.44^{\circ}$ & \\
\hline$A s(1)-S(3)-A s(2)$ & 107.4 & $A s(4)-S(8)-A s(3)$ & 99.5 \\
\hline$A s(2)-S(7)-A s(4)$ & 98.5 & $\begin{array}{r}\mathrm{As}(3)-\mathrm{S}(10)-\mathrm{As}(5 b) \\
\sigma \fallingdotseq 0.40^{\circ}\end{array}$ & 92.6 \\
\hline
\end{tabular}

well with the normal As-S covalent-bond distance. The S-As-S and As-S-As angles are in a good agreement with the values found in the structure of orpiment (N. MoRIмоTо, 1954). As(3) is coordinated

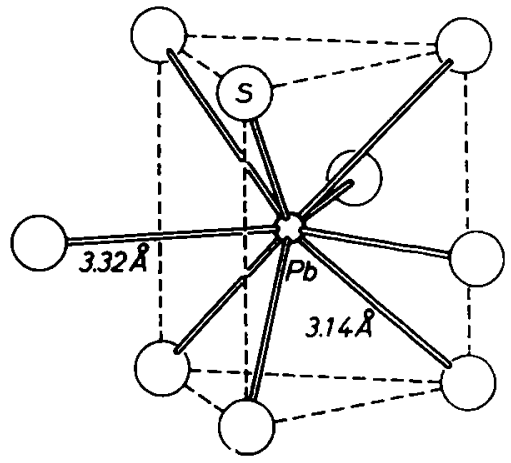

Fig. 2. The configuration of nine $\mathrm{S}$ atoms around a $\mathrm{Pb}$ atom

by $S(5)$ and $S(10)$ at distances of about $2.28 \AA$ and by $S(8)$ and $S(9)$ at distances of about $2.7 \AA$. Although the former are in good agreement with the normal As $-S$ covalent-bond distance, the distances of $2.7 \AA$ are too long for As-S covalent bonds. The magnitude and anisotropy of the temperature motion of $\mathrm{As}(3)$ are very large in comparison to those of $\mathrm{As}(1), \mathrm{As}(2)$ and $\mathrm{As}(4)$, which have a maximum r.m.s. deviation of $0.27 \AA$ and a minimum deviation of $0.18 \AA$ (Table 5). The $A s(3), S(8)$ and $S(9)$ atoms are nearly on a straight line, and As(3) has the largest r.m.s. deviation nearly parallel to this line. Therefore, As(3) seems to form covalent bonds statistically with 
Table 5. The r.m.s. deviations of the atomic positions along the principal axes of the vibration ellipsoids and the direction cosines of the axes refered to the orthogonal axes $X / /$ to $a^{*}, Y / /$ to $b$ and $Z / /$ to $c$

\begin{tabular}{|c|c|c|c|c|}
\hline & r.m.s.d. & $l$ & $m$ & $n$ \\
\hline $\mathrm{Pb}(1)$ & $\begin{array}{l}0.223 \AA \\
0.188 \\
0.212\end{array}$ & $\begin{array}{l}-0.946 \\
-0.060\end{array}$ & $\begin{array}{l}0.285 \\
0.271\end{array}$ & $\begin{array}{r}0.143 \\
-0.959\end{array}$ \\
\hline $\mathrm{Pb}(2)$ & $\begin{array}{l}0.309 \\
0.183 \\
0.227\end{array}$ & $\begin{array}{r}-0.590 \\
-0.050\end{array}$ & $\begin{array}{l}0.678 \\
0.510\end{array}$ & $\begin{array}{r}0.438 \\
-0.858\end{array}$ \\
\hline $\mathrm{Pb}(3)$ & $\begin{array}{l}0.243 \\
0.188 \\
0.216\end{array}$ & $\begin{array}{l}0.332 \\
0.217\end{array}$ & $\begin{array}{l}0.804 \\
0.444\end{array}$ & $\begin{array}{r}0.494 \\
-0.869\end{array}$ \\
\hline $\mathrm{As}(1)$ & $\begin{array}{l}0.190 \\
0.156 \\
0.182\end{array}$ & $\begin{array}{r}0.081 \\
-0.442\end{array}$ & $\begin{array}{l}0.996 \\
0.058\end{array}$ & $\begin{array}{r}-0.023 \\
0.896\end{array}$ \\
\hline $\mathrm{As}(2)$ & $\begin{array}{l}0.212 \\
0.164 \\
0.176\end{array}$ & $\begin{array}{r}0.465 \\
-0.719\end{array}$ & $\begin{array}{l}0.514 \\
0.694\end{array}$ & $\begin{array}{r}0.721 \\
-0.030\end{array}$ \\
\hline $\mathrm{As}(3)$ & $\begin{array}{l}0.271 \\
0.175 \\
0.202\end{array}$ & $\begin{array}{r}0.603 \\
-0.791\end{array}$ & $\begin{array}{l}0.342 \\
0.153\end{array}$ & $\begin{array}{l}0.721 \\
0.588\end{array}$ \\
\hline As(4) & $\begin{array}{l}0.195 \\
0.162 \\
0.175\end{array}$ & $\begin{array}{r}-0.192 \\
0.317\end{array}$ & $\begin{array}{l}0.981 \\
0.035\end{array}$ & $\begin{array}{l}-0.028 \\
-0.949\end{array}$ \\
\hline $\mathbf{A s}(5 a)$ & $\begin{array}{l}0.261 \\
0.215 \\
0.218\end{array}$ & $\begin{array}{l}0.051 \\
0.280\end{array}$ & $\begin{array}{l}0.926 \\
0.354\end{array}$ & $\begin{array}{r}\mathbf{0 . 3 8 3} \\
-\mathbf{0 . 8 9 3}\end{array}$ \\
\hline$A s(5 b)$ & $\begin{array}{l}0.207 \\
0.115 \\
0.179\end{array}$ & $\begin{array}{r}0.004 \\
-0.938\end{array}$ & $\begin{array}{l}0.943 \\
0.124\end{array}$ & $\begin{array}{r}-0.333 \\
\mathbf{0 . 3 2 4}\end{array}$ \\
\hline$S(1)$ & $\begin{array}{l}0.191 \\
0.153 \\
0.170\end{array}$ & $\begin{array}{r}0.823 \\
-0.372\end{array}$ & $\begin{array}{l}0.464 \\
0.875\end{array}$ & $\begin{array}{r}0.325 \\
-0.310\end{array}$ \\
\hline$S(2)$ & $\begin{array}{l}0.178 \\
0.152 \\
0.168\end{array}$ & $\begin{array}{r}0.641 \\
-0.749\end{array}$ & $\begin{array}{l}0.752 \\
0.570\end{array}$ & $\begin{array}{l}0.150 \\
0.340\end{array}$ \\
\hline $\mathbf{S}(3)$ & $\begin{array}{l}0.182 \\
0.138 \\
0.160\end{array}$ & $\begin{array}{r}-0.951 \\
0.042\end{array}$ & $\begin{array}{l}0.209 \\
0.819\end{array}$ & $\begin{array}{r}-0.221 \\
0.572\end{array}$ \\
\hline
\end{tabular}


Table 5. (Continued)

\begin{tabular}{|c|c|c|c|c|}
\hline & r.m.s.d. & $l$ & $m$ & $n$ \\
\hline$S(4)$ & $\begin{array}{l}0.203 \\
0.151 \\
0.155\end{array}$ & $\begin{array}{l}-0.746 \\
-0.268\end{array}$ & $\begin{array}{l}0.347 \\
0.626\end{array}$ & $\begin{array}{r}-0.569 \\
0.733\end{array}$ \\
\hline $\mathbf{S}(5)$ & $\begin{array}{l}0.176 \\
0.145 \\
0.167\end{array}$ & $\begin{array}{r}-0.844 \\
0.361\end{array}$ & $\begin{array}{l}0.492 \\
0.817\end{array}$ & $\begin{array}{r}0.215 \\
-0.450\end{array}$ \\
\hline$S(6)$ & $\begin{array}{l}0.190 \\
0.145 \\
0.173\end{array}$ & $\begin{array}{r}0.930 \\
-0.279\end{array}$ & $\begin{array}{l}0.185 \\
0.918\end{array}$ & $\begin{array}{l}-0.317 \\
-0.283\end{array}$ \\
\hline$S(7)$ & $\begin{array}{l}0.183 \\
0.140 \\
0.173\end{array}$ & $\begin{array}{r}-0.832 \\
0.307\end{array}$ & $\begin{array}{l}0.542 \\
0.258\end{array}$ & $\begin{array}{l}-0.120 \\
-0.917\end{array}$ \\
\hline $\mathbf{S}(\mathbf{8})$ & $\begin{array}{l}0.235 \\
0.150 \\
0.170\end{array}$ & $\begin{array}{l}0.963 \\
0.168\end{array}$ & $\begin{array}{l}0.070 \\
0.597\end{array}$ & $\begin{array}{r}0.259 \\
-0.785\end{array}$ \\
\hline $\mathbf{S}(9)$ & $\begin{array}{l}0.237 \\
0.177 \\
0.195\end{array}$ & $\begin{array}{l}0.938 \\
0.132\end{array}$ & $\begin{array}{l}0.322 \\
0.033\end{array}$ & $\begin{array}{r}-0.136 \\
0.990\end{array}$ \\
\hline$S(10)$ & $\begin{array}{l}0.200 \\
0.155 \\
0.178\end{array}$ & $\begin{array}{r}0.082 \\
-0.448\end{array}$ & $\begin{array}{l}0.869 \\
0.471\end{array}$ & $\begin{array}{r}-0.489 \\
0.761\end{array}$ \\
\hline
\end{tabular}

$\mathrm{S}(8)$ and $\mathrm{S}(9)$. If $\mathrm{As}(3)$ forms a covalent bond with $\mathrm{S}(8)$ the $\mathrm{As}(3)-\mathrm{S}_{3}$ trigonal pyramid is joined with the $\mathrm{As}(4)-\mathrm{S}_{3}$ pyramid.

As(5) was statistically distributed over two positions, $(a)$ and (b), during the course of the refinement. The position $(a)$ is surrounded octahedrally by six $\mathrm{S}$ atoms, while the position $(b)$ has a trigonal pyramidal coordination of three $S$ atoms, which is usual in crystal structures of arsenosulfides. It is suspected that the position $(a)$ is not occupied by As but by a different kind of atom, since the distances from the position $(a)$ to the surrounding $\mathrm{S}$ atoms are too long for As-S distances, and since the sum of the population factors for the positions $(a)$ and $(b)$, as obtained by the least-squares method, is much larger than one. Actually, a careful chemical analysis of the crystal used, carried out by G. BURRI with a CAMECA x-ray microanalyser, showed that the crystal contains a few weight percent of Ag. If the positions (a) are occupied by $\mathrm{Ag}$ atoms, the population factor for (a) becomes about 0.57 and the sum is nearly equal to one. Therefore, 


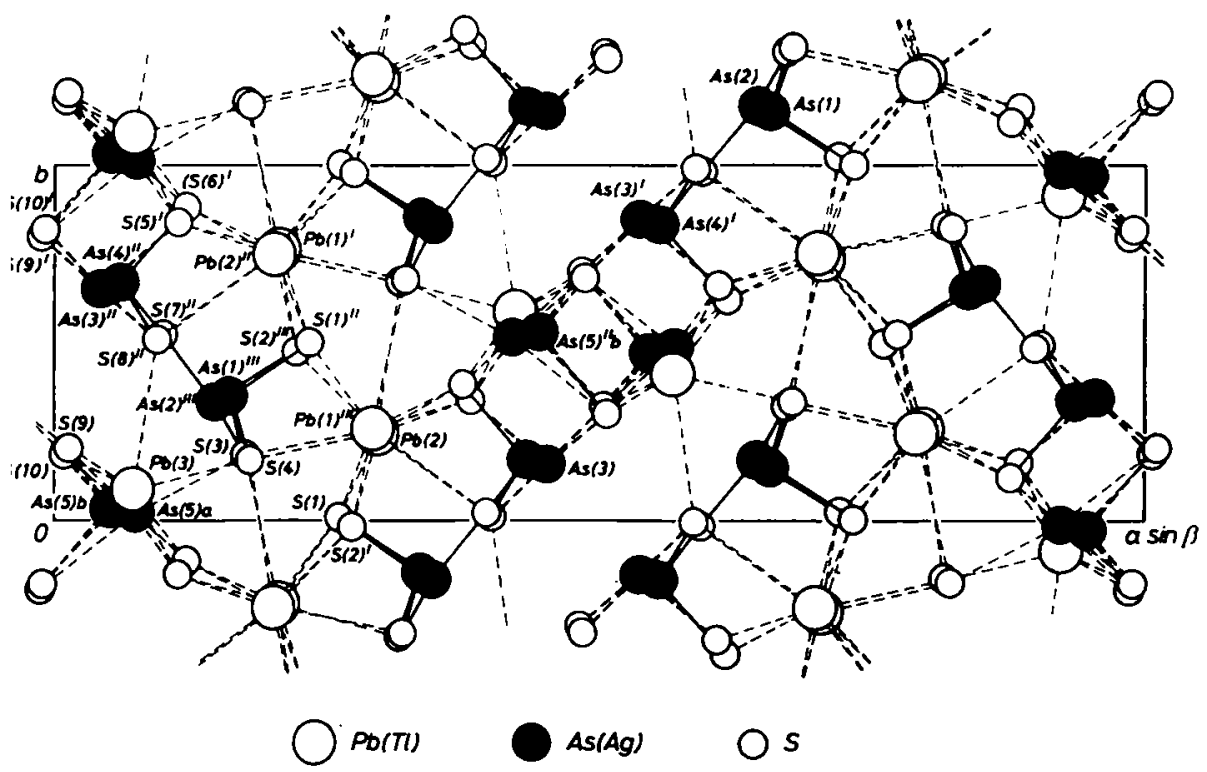

(a)

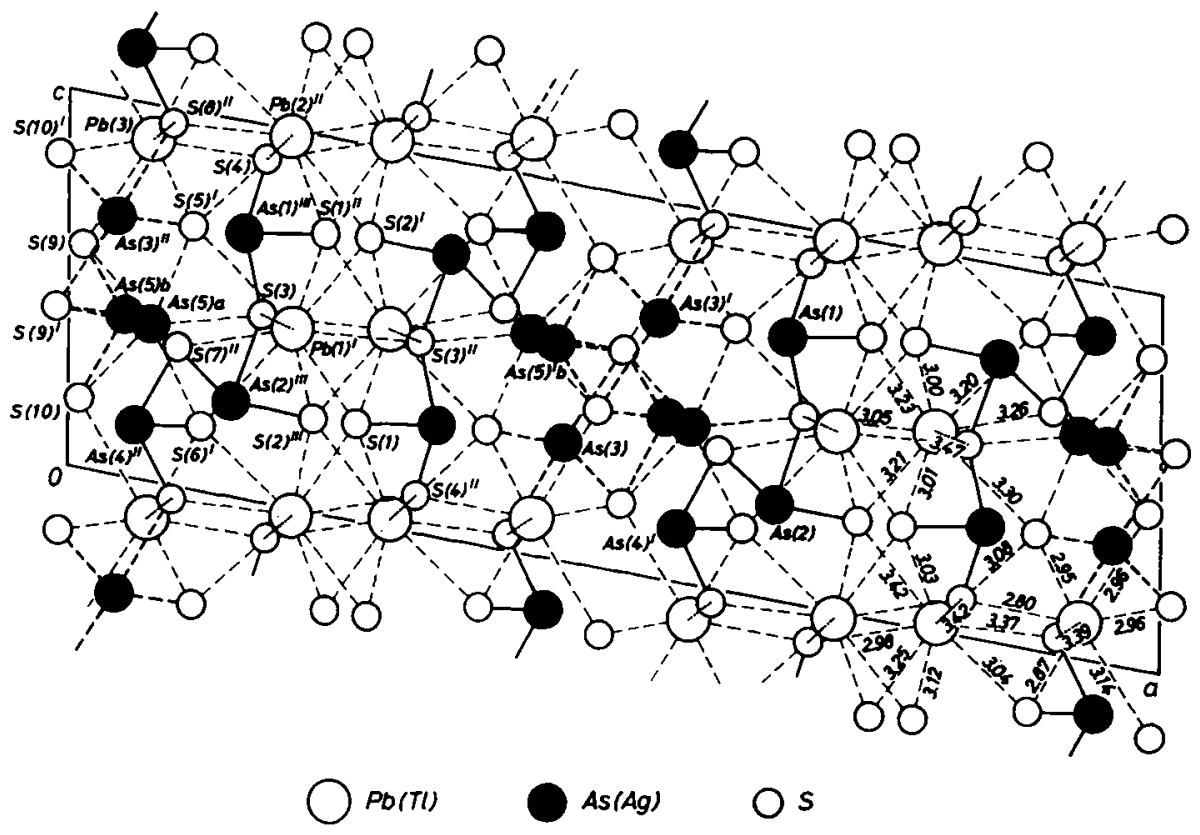

(b)

Fig. 3. The projection of the structure $(a)$ along the $c$ axis and $(b)$ along the $b$ axis 
the position (a) is probably occupied by Ag instead of As. I is not to be expected from the crystallochemical point of view that the As(5) atoms occupy all the $(b)$ positions, since two As(5) $-S_{3}$ trigonal pyramids around a center of symmetry should share two $S$ atoms if it occurs.

The projections of the structure along the $b$ and $c$ axis are shown in Fig. 3(a) and $(b)$. The structure is composed of two kinds of layers parallel to (100). The first kind are the $\mathrm{PbS}_{3}$ layers. The second kind have a structure closely related to the $\mathrm{PbS}$ structure. It is derived from the $\mathrm{PbS}$ structure by dividing it into layers which have the
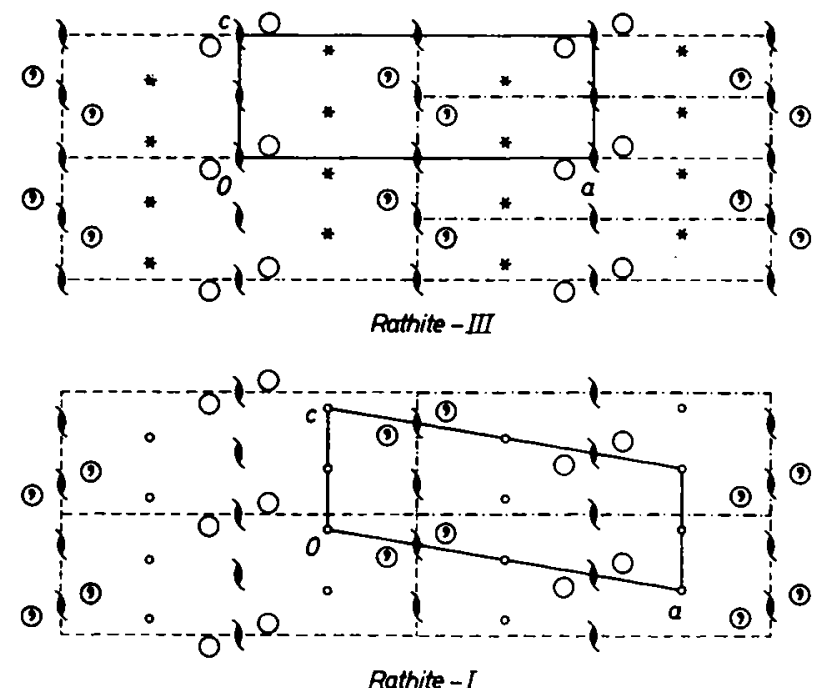

Fig. 4. A comparison of the unit cells and the symmetries of rathite-I and rathite-III. The local centres of symmetry in rathite-III are represented by asteriks. Both of the structures are composed of identical units bounded by the dashed and dotted lines

thickness of $a(\mathrm{PbS})$ and are parallel to (100) of $\mathrm{PbS}$, and by mutually shifting the layers in the [011] direction of PbS by a distance amounting to $a(\mathrm{PbS}) / 2 \sqrt{2}$. The layers in the rathite-I structure correspond to a zone bounded by two planes perpendicular to the [223] direction in the deformed $\mathrm{PbS}$ structure. Although each metallic atom in the deformed $\mathrm{PbS}$ structure is coordinated by seven $\mathrm{S}$ atoms, the As atoms in the rathite-I structure are coordinated by less than seven $\mathrm{S}$ atoms, owing to the fairly large deviation from the ideal atomic configuration caused by the difference in chemical character of As and $\mathrm{Pb}$. 
The main difference in the structure of rathite-I as compared to that of rathite-III (M.-Th. LE Bihan, 1962) lies in the relative positions of $\mathrm{Pb}(3)$ and $\mathrm{As}(5)$. They are made up of the same structural unit, which has the volume of one unit cell (Fig.4). In rathite-III, $\mathrm{Pb}(3)$ and $\mathrm{As}(5)$ are exchanged in the next structural unit along the $a$-axis direction whereby the centre of symmetry which exists in the rathite-I structure is destroyed.

The crystal structures of rathite-II (M.-TH. LE BrHAN, 1962), dufrenoysite (W. NowACKI, F. MARUMO and Y. TAKÉUCHI, 1964), baumhauerite (M.-TH. LE BIHAN, 1962) and scleroclase (W. NowACKI, Y. IrTAKA, H. BÜRKI and V. KUNz, 1961) are also composed of $\mathrm{PbS}_{3}$ layers and layers which have the deformed $\mathrm{PbS}$ structure. The differences between these structures lie in the chemical composition and in the thickness of the second kind of layers.

Although infinite chains of $\mathrm{As}^{-\mathrm{S}_{3}}$ pyramids have been described in the structures of rathite-II, rathite-III and baumhauerite, it is impossible to adapt such chains to the $\mathrm{PbS}_{3}$ layers, as has been pointed out by Y. ItTaka and W. Nowacki (1961) and by Y. Takfuchi, S. Ghose and W. NowACKI (1965). In the strueture of rathite-I the $\mathrm{As}_{\mathrm{S}} \mathrm{S}_{3}$ pyramids form chains with finite lengths. The length of the chain is not fixed since there are several possibilities for the coordinations around the $A s(3)$ and $A s(5)$ atoms as explained above. In the most favourable case, the chain can contain six As- $\mathrm{S}_{3}$ pyramids, in the order of $\mathrm{As}(1)-\mathrm{As}(2)-\mathrm{As}\left(4^{\prime}\right)-\mathrm{As}\left(3^{\prime}\right)-\mathrm{As}\left(5^{\prime \prime}\right)-\mathrm{As}(3)$.

$\mathrm{Tl}$ atoms are thought to be situated at the $\mathrm{Pb}$ position, replacing $\mathrm{Pb}$ atoms. It is not known whether the $\mathrm{Tl}$ atoms are in an ordered state or whether they are statistically distributed over several positions. Probable positions are the $\mathrm{Pb}(2)$ positions, since $\mathrm{Pb}(2)$ has a much larger anisotropic temperature factor than $\mathrm{Pb}(1)$ and $\mathrm{Pb}(3)$.

\section{Acknowledgements}

We thank Prof. L. G. BerRY (Kingston) for the untwinned rathite-I crystal, Prof. W. NEF and Dr. G. Hösser for the possibility of using the Bull $\Gamma$ AET-computer, Dr. J.S. RouLETT of Oxford University Computing Laboratory for calculations on the Mercury computer, the International Business Machines, Extension Suisse, and Cern (Geneva) for the IBM 7090-computer time, Dr. H. BüRKI and Mr. V. KUNz for their help in the $x$-ray experiments and in the calculations by the Bull $\Gamma$ AET computer, Prof. B. J. Wuensor (Cambridge, Mass.) for having introduced the Prewitt least-square refine- 
ment programme, Dr. N. D. Jones (Bern) for his help in improving the English of this paper and Mr. G. BURRI for an additional examination of the rathite-I crystal with the $x$-ray microanalyser. This investigation was sponsored by Schweizerischer Nationalfonds, Kommission zur Förderung der wissenschaftlichen Forschung and Stiftung Entwicklungsfonds Seltene Metalle.

\section{Rolerences}

L. G. Berry (1953), New data on lead sulpharsenides from Binnental, Switzerland. Amer. Min. 88, 330.

Y. IrTAKA and W. Now $A C K I$ (1961), A refinement of the pseudo crystal structure of scleroclase $\mathrm{PbAs}_{2} \mathrm{~S}_{4}$. Acta Crystallogr. 14, 1291-1292.

M.-TH. Le BrHan (1962), Etudo structurale de quelques sulfures de plomb et d'arsénic naturels du gisement de Binn. Bull. Soc. Franç. Min. Cristallogr. 85, $15-47$.

N. Monmoto (1954), The crystal structure of orpiment $\left(\mathrm{As}_{2} \mathrm{~S}_{3}\right)$ refined. Mineral. Journal (Japan) 1, 160-169.

W. NowaCkI und C. Bahrzare (1963), Die Bestimmung der chemischen Zusammensetzung einiger Sulfosalze aus dem Lengenbach (Binnatal, Kt. Wallis) mit Hilfe der elektronischen Mikrosonde. Schweiz. Min. Petr. Mitt. 43, 407-411 (Parker-Festschrift).

W. NowACKI, Y. IrTAKA, H. BÜRKI and V. KUNZ (1961), Structural investigation on sulfoselts from the Lengenbach, Binn Valley (Ct. Wallis). Part 2. Schweiz. Min. Petr. Mitt. 41, 103-116.

W. Nowacki, F. Marumo und Y. TakédChI (1964), Untersuchungen an Sulfiden aus dem Binnatal (Kt. Wallis, Schweiz). Schweiz. Min. Petr. Mitt. 44, 5-9.

M. A. Peacock and L. G. Berky (1940), Röntgenographic observations on ore minerals. Univ. Toronto Studies, Geol. Ser. No. 44, p. 63.

Y. TAKfUOHI, S. GHOBE and W. NOWACKI (1965), The crystal structure of hutchinsonite, $(\mathrm{Tl}, \mathrm{Pb})_{2} \mathrm{As}_{5} \mathrm{~S}_{9}$. Z. Kristallogr. 121, 321-348. 